\title{
DIFUSIVIDADE DA AGUA DO SOLO EM FUNÇÃO DA UMIDADE E DO TEMPO
}

\author{
IVAN AMARAL GUERRINI
}

Orientador: Epaminondas S. B. Ferraz

Dissertação apresentada à Escola Superior de
Agricultura "Luiz de Queiroz" da Universidade
de São Paulo, para obtenção do título de
Mestre em Energia Nuclear na Agricultura.

PIRACICABA

Estado de São Paulo - Brasil

Abril - 1976 
. I.

"Quem luta por uma vida melhor para os outros, acaba fazendo um mundo melhor para si mesmo".

- o Poder da Mensagem (Hézio Ribeiro) 
.II.

dedicado

$\bar{a}$

Virgem Maria 
. III.

Agradecimento Especial aos meus pais e irmãos, cujo amor eleva-me a cada instante $e$ desabrocha em trabalhos como este. 


\section{AGRADECIMENTOS}

Agradeço

- ao Prof. Dr. Epaminondas Sansigolo de Barros Ferraz, que me acompanhou nos primeiros passos dentro da pesquisa,sem pre me apoiando com uma orientação segura, a qual foi mui to valiosa na confecção deste trabalho;

- ao Prof. Dr. Klaus Reichardt, exemplo de amigo e conseIheiro, sempre disponivel e pronto a colaborar;

- ao Pe. João Modesti, modelo de professor, em cujas aulas aprendi que gostar de física é também um dom de Deus;

- ao meu tio Antonio Kerches de Campos, que não mediu esfor ços para me ajudar;

- aos meus amigos do movimento jovem D.D.V., cuja companhia sempre foi um incentivo a trabalhar com amor;

- à colega, e agora amiga, Eliane Nogueira de Queiroz, com quem tive prazer imenso de trabalhar;

- aos colegas João Antonio Siqueira Neto, Sandra Voltani Queiroz e Paulo Leonel Libardi, pela amizade e pelas várias sugestões por demais importantes;

- aos funcionários Iolanda Aparecida Rufini, Valdir Antonio Ximenes e ao desenhista Antonio Aparecido Martins, pela preciosa colaboração; 
.$v$.

- à datilografa Sónia Novaes Rasera e aos funcionários Bene dito Herculano Davanzo e Luis Cesar Zambello, pelos serví ços prestados;

- à Comissão Nacional de Energia Nuclear, pela bolsa de estudos concedida;

- a todos quanto tem participação neste trabalho. 


\section{INDICE}

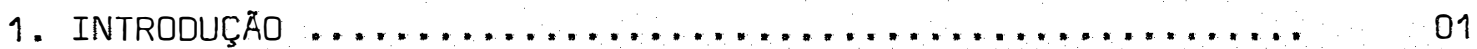

2. REVISÃO BIBLIOGRĀFICA $\ldots \ldots \ldots \ldots \ldots \ldots \ldots \ldots \ldots \ldots \ldots \ldots \ldots \ldots \ldots$

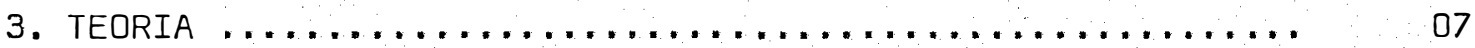

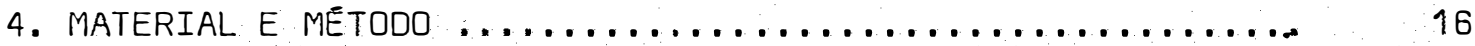

4.1. Solos Utilizados $\ldots \ldots \ldots \ldots \ldots \ldots \ldots \ldots \ldots \ldots \ldots \ldots \ldots . . \ldots$

4.2. Colunas de Solo ....................... 17

4.3. Metodologia...$\ldots \ldots \ldots \ldots \ldots \ldots \ldots \ldots \ldots \ldots \ldots \ldots .20$

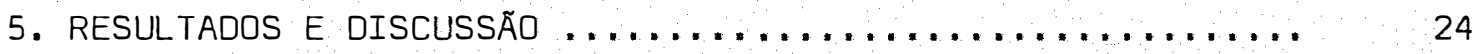

5.1. Obtenção de alguns coeficientes .............. 24

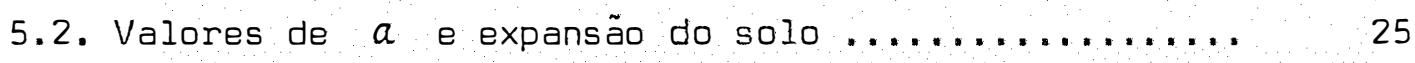

5.3. Perfis de umidade e difusividade .............. 27

5.4. Liberdade de expansão para os solos ............. 28

5.5. Método e arranjo experimental ................. 28

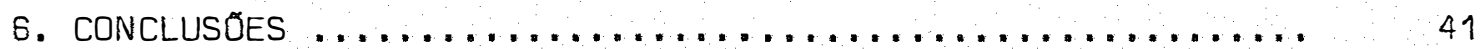

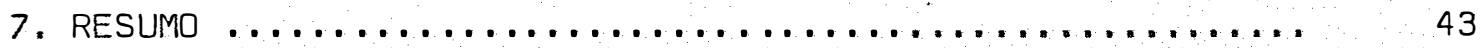

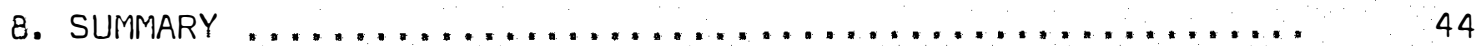

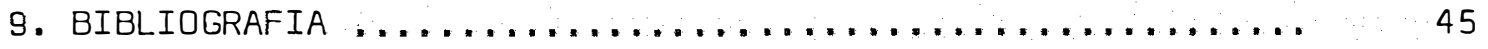


I. Análise mecánica das amostras de solo utilizadas ......

II. Valores de densidade e umidade e seus respectivos erros

III. Valores dos coeficientes de cada amostra, necessários à

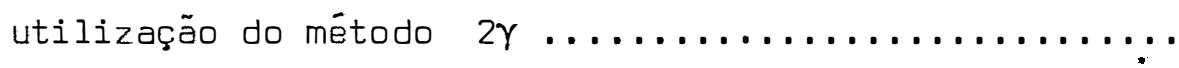

IV. Valores do parámetro $a$, do coeficiente de correlação da reta (32), e da expansão total da amostra de solo...

V. Valores de difusividades para a amostra $1 \ldots \ldots \ldots \ldots \ldots$

VI. Valores de difusividades para as amostras 2 e 3, os quais não variam no tempo, e portanto, $D(\theta, t) \equiv D_{1}(\theta)$ $\equiv D^{*}(\theta)$, já que essas amostras apresentaram $a=0,50$.

VII. Valores de difusividades para a amostra $4 \ldots \ldots \ldots \ldots$. 


\section{LISTA DE FIGURAS}

no

I. Coluna de solo com os dispositivos utilizados .........

II. Retas referentes à equação (32) onde aparecem os dados

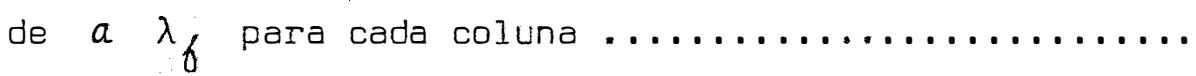

III. Perfis de umidade para a amostra $1 \ldots \ldots \ldots \ldots \ldots \ldots \ldots$

IV. Perfis de umidade para a amostra $2 \ldots \ldots \ldots \ldots \ldots \ldots$

V. Perfis de umidade para a amostra $3 \ldots \ldots \ldots \ldots \ldots \ldots \ldots$

VI. Perfis de umidade para a amostra $4 \ldots \ldots \ldots \ldots \ldots \ldots \ldots$

VII. Perfis de umidade para a amostra $5 \ldots \ldots \ldots \ldots \ldots \ldots \ldots$

VIII.Razão entre as densidades referentes aos valores de umidades correspondentes e a densidade média da coluna se-

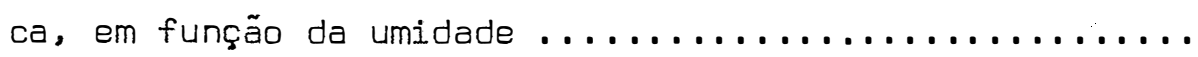




\section{INTRODUÇAOO}

No estudo do movimento da água no solo, uma das funções mais importantes a ser determinada é a difusividade. No caso do movimento horizontal, a equação geral é mais simples, devido a auséncia do potencial gravitacional, e, portanto, a difusividade é mais fa cilmente calculada.

Durante a infiltração da água no solo, é de se esperar que ociorra, ao menos, um pequeno rearranjo das partículas deste à pas sagem da água, e, em alguns casos, até uma expansão do solo com conse quente diminuição de sua densidade no ponto em questão. Naturalmente, tais fatos exigem que a difusividade não dependa apenas da umidade do solo, mas também da posição considerada, já que um rearranjo das par- 
tículas atua como uma modificação na estrutura do sistema. No entanto, os estudos feitos até hoje tem ignorado, na maloria das vezes, es sa dependéncia da posição, mais por conveniéncia matemática, è bem verdade.

$$
\text { Este trabalho, propõe a hipótese dessa dependéncia, }
$$
transformando-a numa dependéncia do tempo, e apresentando, não somente uma solução para o caso de infiltração da ägua em solos expansivos, como também uma nova solução matemática para problemas de infiltração da água no solo, semelhante à utilizada até agora e com resultados experimentais que a confirmam. 
.3.

\section{REVISÃO BIBLIOGRĀFICA}

No estudo do movimento da ägua no solo, entre tantos aspectos importantes, dois deles tem preocupado, de maneira marcante, os pesquisadores no assunto. Um deles é o problema de infiltração em solos que se expandem, e o outro é um melhor conhecimento da função difusividade da água do solo.

Com relação ao movimento da água em solos expansivos, tem sido desenvolvido, recentemente, muita teoria, sendo obtidos poucos resultados práticos satisfatórios. Alguns dos trabalhos iniciais foram os de FOX (1964) e ZASLAVSKY (1964) os quais apresentam uma anā lise do problema do ponto de vista euleriano, o qual preserva uma conecção direta entre a física e a matemática do processo, sendo que es 
ta última, no entanto, torna-se bastante complicada. Nessa análise, o sistema de coordenadas permanece fixo.

Depois desses, surgiram alguns outros trabalhos no mes mo sentido, de JONG (1966) e o de EL-SWAIFY e HENDERSON (1967) até que SMILES e ROSENTHAL (1968) propuseram uma nova maneira de abordar - assunto, através da análise lagrangiana. Esta tem por fundamento uma matemática elegante e simplificada, a qual, no entanto, não tem uma conecção imediata com a física. Nesta análise, o sistema de coor denadas está preso às partículas do solo, o que implica que estas sem pre estarão em repouso, mesmo que haja rearranjo.

PHILIP (1968) mostrou a mesma análise num estudo semelhante, sobre variação de volume de solos argilosos quando da passagem da água. Num trabalho posterior, PHILIP (1969a) fez um resumo bastante didático da teoria de infiltração da água em solos expansivos, reunindo as idéias de SMILES e ROSENTHAL (1968) e do próprio PHI LIP (1968). BRIONES e UEHARA (1969) utilizando-se da mesma análise: apresentam um importante trabalho com solos que se contraem.

Outra contribuição importante, reforçando ainda mais a análise lagrangiana, é a de PHILIP e SMILES (1969) estudanto variação de volume em sistemas que apresentam três componentes: solo, água e ar.

Em dois trabalhos subsequentes, PHILIP (1969b e e) pra ticamente iniciou o estudo do movimento da água, na vertical, em solos expansivos, evidenciando a importáncia do potencial "overburden " e apresentando dados bastante interessantes sobre o caráter expansivo dos solos analisados.

Para os estudos de laboratório, especificamente, há uma publicação de LAL et alii (1970) que diz respeito ao diámetro das colunas de solo, utilizadas para infiltração em solos expansivos. De ve ser ressaltado também, os trabalhos de COLLIS-GEORGE e LAL (1970) e BRIDGE et alii (1970) que tratam de melhoramentos em colunas des- 
se tipo.

Estudos semelhantes e dignos de serem mencionados, são os de SMILES e POULOS (1969), PHILIP (1970), COLLIS-GEORGE e BRIDGE (1973), de COUGHLAN et alii (1973) e o de BRIDGE e COLLIS-GEORGE $(1973 a)$.

Bem recentemente, SMILES e FARVEY (1973) apresentaram um trabalio de complementação daquele publicado por SMILES e ROSENTHAL (1968), Utilizando sempre a mesma análise lagrangiana.

Devem ser citadas tambēm, as recentes contribuições de TALSMA (1974) e PARLANGE (1975a), onde predomina, no entanto, uma difícil interpretação dos resultados.

A maioria dos pesquisadores de física da água do solo, no que diz respeito a solos expansivos, atualmente ainda dão preferén cia à análise lagrangiana, apesar da dificuldade, já mencionada, de não haver ligação imediata e evidente entre a física e a matemática do fenômeno. Alguns como DOLEZAL e KUTILEK. (1972) e YONG e WARKENTIN (1972), preservam a análise de Euler, apresentando, porém, uma matemá tica muito complicada.

De grande importância também são os trabalhos de BRIDGE e COLIIS-GEORGE (19733) e o de STROOSNIJDER e SWART (1974), os quais referem-se a infiltração da água em solos expansivos, utilizando o método $2 \gamma$, com o qual se determina, simultaneamente, densidade e umidade do solo, ponto por ponto, numa coluna. Esse método é explica do com detalhes por FERRAZ (1974) e é, atualmente, o único que permite um estudo bem feito do movimento da água em solos que apresentam alguma expansão ou apenas rearranjo interno das partículas, como $\bar{\varepsilon}$ mostrado por FERRAZ e GUERRINI (1975).

Por outro lado, pesquisas estão em andamento a busca de um melhor entendimento da função difusividade da água do solo,quan to a. sua dependéncia com a umidade. Assim, por exemplo, AHUJA e 
SWARTZENDRUBER (1972) mostram a difusividade como uma função potência, do tipo infinito. Já REICHARDT et alii (1972) apresentam, a par tir do conceito de meio similar, uma generalização da teoria de infil tração, no que diz respeito à obtenção da difusividade. Esta pode ser obtida, segundo REICHARDT e LIBARDI (1973), apenas através da taxa de variação da posição da frente de molhamento com o tempo. Nesses trabalhos, aparece a difusividade como função exponencial da umidade. Es sa dependència pode ser observada também em PARLANGE (1975b), onde o autor apresenta uma análise teórica de infiltração horizontal em solos insaturados.

Já LAROUSSI et alii (1975) mostram que essa variação é exponencial apenas num determinado intervalo, sendo inclusive decrescente com a umidade para valores baixos desta.

Na verdade, a suposição de que a difusividade é apenas função da umidade, é um fator limitante na qualidade dos resultados até então obtidos. É certo que, se fosse levada avante a hipótese da dependéncia da posição para a difusividade, os resultados seriam bem melhores, muito embora, em princípio, a teoria seria bem mais complicada. O que parece ter fugido aos olhos dos pesquisadores, é o fato dessa dependéncia da posição poder vir a resolver tanto o problema da função difusividade, como também o problema de infiltração da água em solos que se expandem.

Nesse sentido, REICHARDT e NIELSEN (1975) deram um grande passo, apresentando uma anälise matemätica relativamente simples, introduzindo a dependéncia do tempo para a difusividade, onde a idéia, no entanto, é apenas apresentar um estudo de infiltração da água em solos expansivos. 
.7.

3. TEORIA

As equações que descrevem o estudo de infiltração da água em colunas de solo homogênẹ, na horizontal, em apenas uma dimen são e em regime não saturado são:
a equação de DARCY
$q_{x}=-\kappa\left(\frac{\partial \xi}{\partial x}\right) \ldots(1)$
a equação da continuidade $\frac{\partial \theta}{\partial t}=\frac{\partial\left(q_{\chi}\right)}{\partial x} \ldots \ldots$ (2)

onde:

$q_{\chi}=$ componente horizontal da densidade de fluxo da 


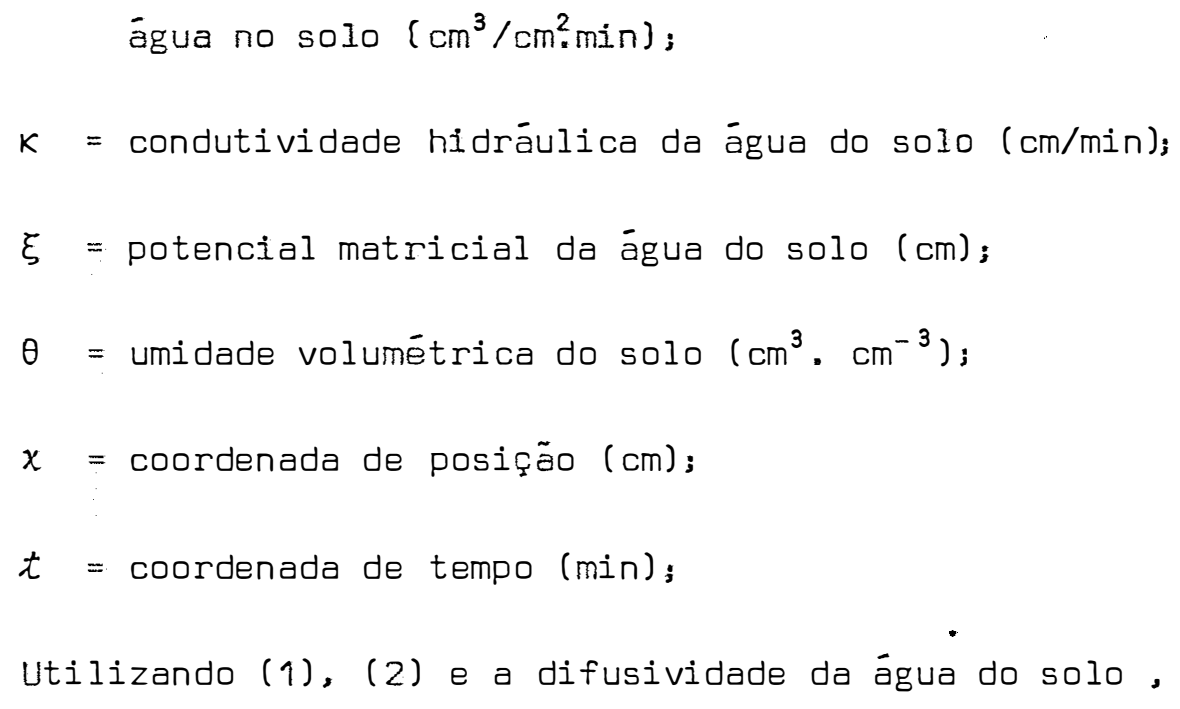

$D \equiv \kappa \frac{\partial \xi}{\partial \theta}$

chega-se à:

$$
\frac{\partial \theta}{\partial t}=\frac{\partial}{\partial x}\left[D \frac{\partial \theta}{\partial x}\right]
$$

que é a equação diferencial mais geral do movimento horizontal da àgua no solo.

A condutividade hidráulica, $K$, é um dos parâmetros mais importantes para estudos de água no solo. Experimentalmente, ve rificou-se que:

$$
\kappa=\frac{\kappa^{\prime} \rho_{e} g}{n}
$$

onde

$$
\begin{aligned}
K^{\prime}= & \text { propriedade do solo chamada permeabizidade in- } \\
& \text { trinseca, que depende da umidade e do arranjo } \\
& \text { geométrico das partículas }\left(\mathrm{cm}^{2}\right) \text {; } \\
\rho_{e}= & \text { densidade do fluido (água) }\left(\mathrm{g} / \mathrm{cm}^{3}\right) ;
\end{aligned}
$$




$$
\begin{aligned}
& g \text { = aceleração da gravidade }\left(\mathrm{cm} / \mathrm{seg}^{2}\right) ; \\
& \eta \text { = viscosidade do fluido }(\mathrm{g} / \mathrm{cm} \cdot \mathrm{seg}) .
\end{aligned}
$$

Nos materiais porosos rígídos, $K^{\prime}$ é função só da umidade e assim também $k$, já que $\rho_{e}, g$ e $n$ permanecem constantes para um dado experimento. Contudo, no estudo de infiltração da ägua no solo, este não se comporta exatamente como um material poroso rígi do. Em alguns casos, a entrada. da água pode provocar um aumento de volume do solo (solos expansivos), e em outros casos, apenas um rearranjo interno de suas partículas. Pode-se assegurar que, ao menos um pequeno rearranjo interno sempre haverá quando da passagem da água no solo, mesmo que não haja expansão. Dessa maneira, para um estudo rigoroso, deve-se considerar $K$ ' não apenas função da umidade, mas também da posição, pois, punto por ponto, as condições se modificam. As sim:

$$
K^{\prime}=\kappa^{\prime}(\theta, x)
$$

condição que leva à:

$$
\kappa=k(\theta, x)
$$

Utilizando-se de (7) e (3) e sabendo-se que o potencial $\xi$ é função apenas de $\theta$, obtem-se:

$$
D=D(\theta, x)
$$

- que, juntamente com a equação (4), resulta em:

$$
\frac{\partial \theta}{\partial t}=\frac{\partial}{\partial x}\left[D(\theta, x) \frac{\partial \theta}{\partial x}\right]
$$

A equação (9), como se apresenta, é muito difícil de ser resolvida. Sabe-se, no entanto, que as três variáveis em jogo,no 
.10

caso de infiltração horizontal da água no solo são: umidade, posição e tempo $(\theta, x, t)$, de tal maneira que pode-se escrever $\theta=\theta(x, t)$ ou também $x=x(\theta, t)$ mantendo sempre, no caso, o tempo como variável independente. Assim sendo, escrever $D=D(\theta, \chi)$ é o mesmo que escrever $D=D(\theta, t)$, e da equação (g) tem-se:

$$
\frac{\partial \theta}{\partial t}=\frac{\partial}{\partial x}\left[D(\theta, t) \frac{\partial \theta}{\partial x}\right]
$$

Uma hipótese conveniente e bastante lógica é a de supor, de acordo com REICHARDT e NIELSEN (1975), que a parte da difusividade que varia com a umidade é independente da parte que varia com o tempo, da seguinte maneira:

$$
\begin{aligned}
& D(\theta, t)=D_{1}(\theta) \cdot D_{2}(t) \\
& D e(10) \text { e }(11) \text { vem: }
\end{aligned}
$$

$$
\frac{\partial \theta}{\partial t}=\frac{\partial}{\partial x}\left[\left(D_{1}(\theta) \cdot D_{2}(t)\right) \frac{\partial \theta}{\partial x}\right]
$$

e como $D_{2}$ é somente função do tempo,

$$
\frac{\partial \theta}{\partial t}=D_{2}(t) \frac{\partial}{\partial x}\left[D_{1}(\theta) \frac{\partial \theta}{\partial x}\right]
$$

e

$$
\frac{1}{D_{2}(t)} \frac{\partial \theta}{\partial t}=\frac{\partial}{\partial x}\left[D_{1}(\theta)-\frac{\partial \theta}{\partial x}\right]
$$

$$
\text { Define-se agora, um parámetro } \Omega \text { tal que: }
$$

$$
\frac{\partial \Omega}{\partial t} \equiv D_{2}(t)
$$


Substituindo-se esse valor de $D_{2}(t)$ na equação (14):

$$
\frac{\partial \theta}{\partial \Omega}=\frac{\partial}{\partial x}\left[D_{1}(\theta) \frac{\partial \theta}{\partial x}\right]
$$

Para resolver (16), SWARTZENDRUBER (1969) sugeriu que $x$ fosse transformada em variável dependente, isto é:

$$
x=\chi(\theta, \Omega)
$$

e assim fazendo, a equação (16) apresenta como solução parcial

$$
\chi=\lambda(\theta) \Omega^{1 / 2}
$$

onde $\lambda(\theta)$ é uma função, em princípio desconhecida, que depende apenas da umidade.

Essa solução nos diz que, na infiltração horizontal, o avanço, $\chi$, de uma mesma umidade, $\theta$, dentro da coluna é proporcional à raiz quadrada do novo parámetro $\Omega$, o que acontece, por exemplo, no avarıço da frente de molhamento, onde a umidade é constante e, portanto, tem-se:

$$
x_{6}=\lambda_{6} \Omega^{1 / 2}
$$

onde $\chi_{f}$ é a posição da frente de molhamento num dado instante e $\lambda_{6}$ é um valor constante correspondente à umidade da frente ${ } f$. Desde que na frente de molhamento a umidade permanece constante, e, sabendo -se que $x=x(\theta, t)$, pode-se escrever que:

$$
\begin{aligned}
& x_{6}=x_{6}(t) \quad \ldots \ldots \ldots \ldots \ldots \ldots \ldots \ldots \ldots \ldots \ldots \ldots \ldots \ldots \ldots \ldots \ldots \\
& \text { Das equações }(19) \text { e }(20) \text {, nota-se que } \Omega \text { deve ser fun }
\end{aligned}
$$
ção do tempo

$$
\Omega=\Omega(t)
$$


Acompanhando, então, experimentalmente, o avanço da frente de molhamento, observa-se que $\chi$ versus $t^{1 / 2}$ nem sempre é a melhor reta, a qual, no entanto, pode ser obtida ajustando um outro expoente, $a$, para o tempo, não necessariamente igual a 0,50 ,

$$
x_{6}=A t^{a}
$$

onde a é um número puro que depende não só do tipo de solo, como também do arranjo interno de suas partículas, e A, uma constante, que, de acordo com (19), deve ser o próprio $\lambda_{f}$. Assim: .

$$
\begin{aligned}
& x_{f}=\lambda_{f} t^{a} \quad \ldots \ldots \ldots \ldots \ldots \ldots \ldots \ldots \ldots \ldots \ldots \ldots \ldots \ldots \ldots \ldots \ldots \\
& \text { Das equaços }(19) \text { e }(23), \text { tem-se: } \\
& \Omega^{1 / 2}=t^{a}
\end{aligned}
$$

ou

$$
\Omega=t^{2 a}
$$

Utilizando-se agora, do valor de $\Omega$ obtido em (24), na equação (15),

$$
D_{2}(t)=\frac{\partial \Omega}{\partial t}=\frac{\partial\left(t^{2} a\right)}{\partial t}
$$

e

$$
D_{2}(t)=2 a t^{(2 a-1)}
$$

A partir da equação (16), BRUCE e KLUTE (1956) apresen tam um método para a determinação da difusividade, num dado instante $t_{0}$. 


$$
D_{1}(\theta)=\frac{1}{2 \Omega_{0}}\left(\frac{-d x}{d \theta}-\right) \int_{\theta_{i}}^{\theta} x d \theta \quad \ldots \ldots \ldots \ldots \ldots(26)
$$

onde $\Omega_{0}$ é o valor de $\Omega$ quando $t=t_{0} ; \theta_{i}$ é o valor inicial da umidade e $\theta$ é o valor da umidade no instante considerado. De (24) e (26), vem:

$$
D_{1}(\theta)=\frac{1}{2 t_{0}^{(2 a)}}\left(\frac{-d x}{d \theta}\right) \int_{\theta_{i}}^{\theta} x d \theta
$$

Nessa equação (27), qualquer valor atribuído a $t_{0}$ le vará a um mesmo $D_{1}$, mostrando que este independe do tempo, ao contrário do que possa parecer à primeira vista, o que é mostrado por BRUCE e KLUTE no trabalho acima citado. Sabe-se também que

$$
D(\theta, t)=D_{1}(\theta) \cdot D_{2}(t)
$$

e portanto:

$$
D(\theta, t)=D_{2}(t) \cdot \frac{1}{2 t_{0}|2 a|}\left(-\frac{-d x}{d \theta}\right) \int_{\theta_{i}}^{\theta} x d \theta
$$

Usando (25) e (28):

$$
D(\theta, t)=\left[2 a t^{(2 a-1)}\right]\left[\frac{1}{2 t_{0}^{(2 a)}}\left(\frac{-d x}{d \theta}-\right) \int_{\theta_{i}}^{\theta} x d \theta\right] \ldots(29)
$$

que é a equação mais geral para a obtenção da difusividade da água do solo, seja este expansivo ou não. Para um dado instante $t_{0}$ então, a difusividade toma a expressão simplificada: 


$$
D\left(\theta, t_{0}\right)=\frac{a}{t_{0}}\left(\frac{-d x}{d \theta}\right) \int_{\theta_{i}}^{\theta} x d \theta \quad \ldots \ldots \ldots \ldots \ldots
$$

que é quase idèntica-à equação

$$
D *(\theta)=\frac{1}{2 t_{0}}\left(\frac{-d x}{d \theta}\right) \int_{\theta_{i}}^{\theta} x d \theta
$$

utilizada por BRUCE e KLUTE considerando a difusividade função apenas da umidade.

Para a determinação do parámetro $a$, resolve-se (23) aplicando-se logaritmo,

$$
\log x_{6}=\log \lambda_{6}+a \log t
$$

e observa-se que a é o coeficiente angular da reta (32) e, portanto, pode ser calculado facilmente.

E de se esperar que, para solos não expansivos ou para os quais o rearranjo interno das partículas seja desprezível, o valor de a seja praticamente 0,50. Nesse caso, ter-se-ia a equação (24):

$$
\Omega=t
$$

mostrando que o novo parámetro $\Omega$ coincidiria com o próprio tempo. Da equação (25) viria:

$$
D_{2}(t)=1
$$

- que mostra que, nesse caso, a difusividade da água do solo não depende do tempo. Utilizando (29) e o valor de a igual a 0,50, obtem -se: 
.15.

$$
D(\theta, t)=D_{1}(\theta)=D *(\theta)=\frac{1}{2 t_{0}}\left(\frac{-d x}{d \theta}\right) \int_{\theta_{i}}^{\theta} x d \theta \ldots
$$

que é exatamente a expressäo de BRUCE e KLUTE comumente utilizada para calculo da difusividade. Deve-se notar, entretanto, que a expressão (35) é válida apenas quando $a=0,50$. Quando a tem outro valor, a expressão correta a ser utilizada é a (29) pois, nesse caso, a difusividade da àgua do solo é função do tempo também, e essa dependência é tanto mais acentuada quanto maior for a diferençà entre $a$ 0,50 . 
.16.

4. MATERIAL E METTODO

\subsection{Solos Utilizados}

Procurou-se usar nos experimentos, solos que se sabia de antemão, terem comportamento expansivo. Assim sendo, os solos uti lizados foram algumas variações do Litossol Substrato Folheto-Argilito (LSFA) da localidade de Lageadinho no município de Piracicaba, Estado de São Paulo e Vertissolo, da localidade de E.E. Mandacaru do mu nicípio de Joazeiro, Estado da Bahia.

Quatro amostras de Litossol foram retiradas de uma man cha de cujo centro foi coletada a Amostra 2, a qual foi tomada como base. As variações utilizadas foram as seguintes: 
Amostra 1: LSFA retirado a $1 \mathrm{~km}$ da base;

Amostra 2: LSFA retirado do centro da mancha;

Amostra 3: LSFA retirado da base, porém contendo pedras, as quais foram moidas;

Amostra 4: LSFA retirado a $10 \mathrm{~m}$ da base;

Amostra 5: Vertissolo do nordeste brasileiro.

Todas as amostras foram retiradas de camadas de 0 a 20 cm de profundidade e apresentaram a análise mecánica, mosṭrada no Qua dro I.

\begin{tabular}{lccc}
\hline & $\begin{array}{c}\text { Areia } \\
(\%)\end{array}$ & $\begin{array}{c}\text { Limo } \\
(\%)\end{array}$ & $\begin{array}{c}\text { Argila } \\
(\%)\end{array}$ \\
\hline $\begin{array}{c}\text { Litossol (Piracicaba) } \\
(\text { amostra 2) }\end{array}$ & 25,7 & 44,0 & 30,3 \\
\hline $\begin{array}{c}\text { Vertissolo (Joazeiro) } \\
\text { (amostra 5) }\end{array}$ & 10,0 & 75,0 & 15,0 \\
\hline
\end{tabular}

Quadro I. Análise mecánica das amostras de solo utilizadas.

\subsection{Colunas de Solo}

As amostras de solo foram secas ao ar, à sombra, por 48 horas e depois peneiradas em peneiras de malha $1 \mathrm{~mm}$. A umidade re sidual inicial foi determinada por pesagens antes e após a permanéncia da amostra por 48 horas, em estufa a $105^{\circ} \mathrm{C}$. 0 solo preparado foi acondicionado, com compactações uniformes, em recipientes cilíndricos de plástico acrílico de $50 \mathrm{~cm}$ de comprimento por $5,65 \mathrm{~cm}$ de diámetro interno, sendo de $0,3 \mathrm{~cm}$ a espessura da parede. A essa montagem dá- se nome de columa. 
feita facilidade de se movimentar dentro da coluna, para medir a expansão do solo durante a infiltração da água. Para tanto, o solo não preencheu todo o espaço da coluna, restando cerca de $6,0 \mathrm{~cm}$ na extremidade de entrada da água, onde o pistão foi introduzido e encostado ao solo, usando-se um papel-filtro como intermediärio. Esse pistão apresenta-se cheio de furos, dando chance a que a água atingisse o so lo por igual, contribuindo para isso o papel-filtro. Foi utilizada também uma mola, adaptada entre a tampa de entrada da coluna e o pistão, para evitar que este se movimentasse apenas com a água. 0 comprimento normal dessa mola é de $5,0 \mathrm{~cm}$ e admitiu-se que ela observasse a lei de Hooke:

$$
F(x)=-k x
$$

onde $F(x)$ é a força de restauração da mola, ocasionada por um deslo camento $\chi$, e $K$ é uma constante característica da mola. A utilização de (36) vem do fato de que os deslocamentos utilizados serem pequenos em comparação com o comprimento normal da mola. A constante $K$ foi calculada em 167,0 gf/cm. Para uma compressão de $0,5 \mathrm{~cm}$, como foi, em média, verificado, resulta uma força de 83,0 gf, considerada insuficiente para conter a expansão do solo, como foi, aliás, observa do. A outra extremidade da coluna foi fechada normalmente, deixando-se apenas alguns orifícios para saída do ar do solo empurrado pela


tremidades da coluna. Todo esse dispositivo pode ser visualizado na Figura I. Para a compactação do solo e problemas de admissão da água na coluna, foram usadas as técnicas adotadas por LIBARDI (1973). 
.19.

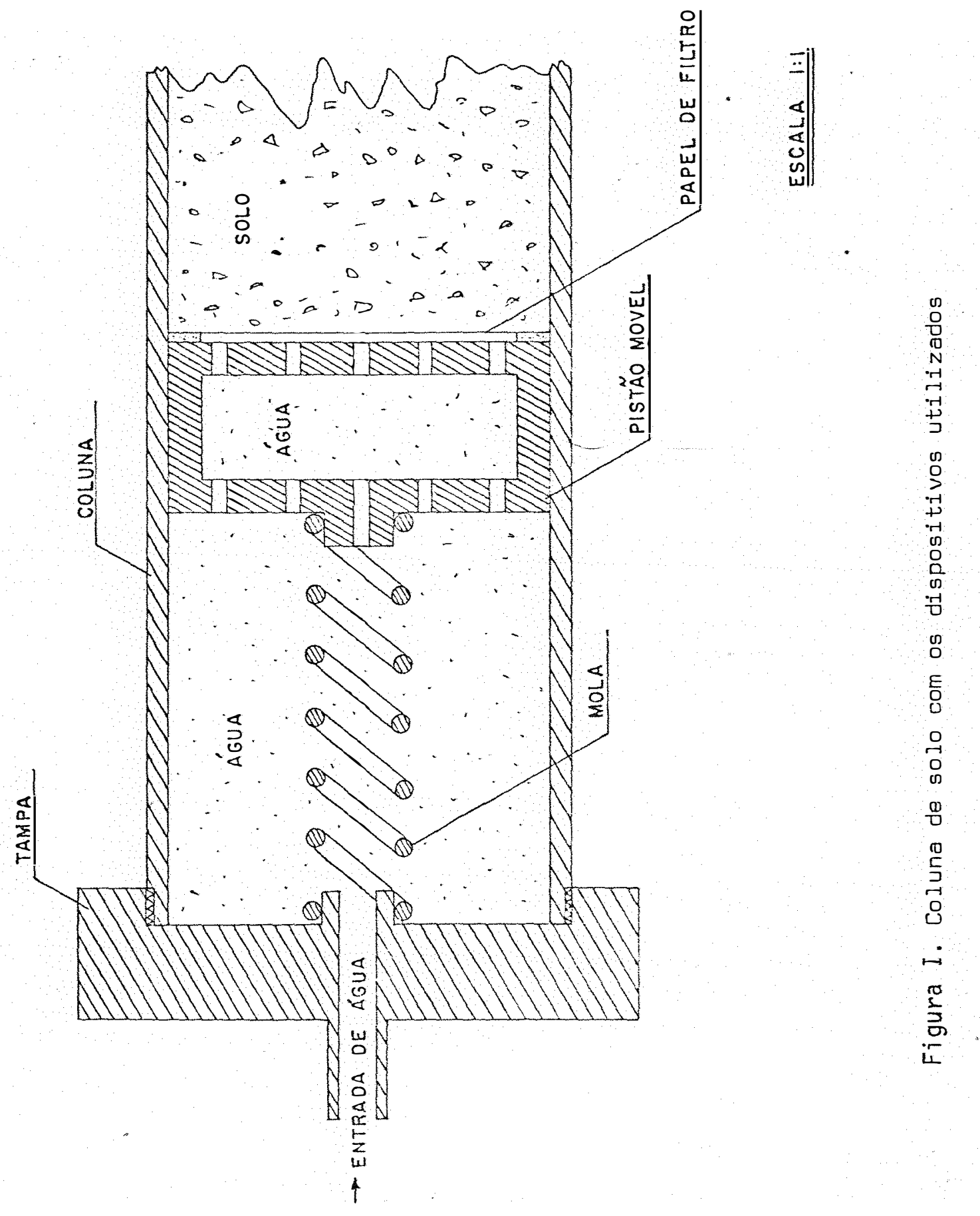


.20.

\subsection{Metodo Togia}

A parte experimental que diz respeito à obtenção dos valores de umidade ao longo da coluna foi realizada com o equipamento e a metodologia desenvolvida por FERRAZ (1974), partindo de uma idéia original de SOANE (1967), denominado Método $2 \gamma$. Este permite a deter minação da densidade, simultaneamente com a umidade, ponto por ponto, na coluna.

Numa geometria paralela, dois feixes colimados, distan tes entre si de $10 \mathrm{~cm}$, sendo um de $60 \mathrm{KeV}$ de energia e outro de 662 KeV de energia, atingem o corpo de prova, são parcialmente absorvidos por este e a fração transmitida é detectada e analisada.

Como fonte de $60 \mathrm{KeV}$, foi usada uma amostra de $100 \mathrm{mCi}$ de ${ }^{241} \mathrm{Am}$, inserida num colimador de chumbo de $12,4 \mathrm{~mm}^{2}$ de secção reta circular. A distáncia de colimação no lado da amostra é de $3,0 \mathrm{~cm}$ e no lado do detector é de $2,0 \mathrm{~cm}$, o que permite um àngulo plano de dis persão inferior a $3^{0}$. Como fonte de $662 \mathrm{KeV}$, foi usada uma amostra de $130 \mathrm{mCi}$ de ${ }^{137} \mathrm{Cs}$, em colimador de chumbo de $4,5 \mathrm{~mm}^{2}$ de secção reta circular. A distáncia de colimação no lado da amostra é de $15,0 \mathrm{~cm}$ e no lado do detector, $2,0 \mathrm{~cm}$, o que proporciona um àngulo de dispersão desprezível.

D detector usado é um cristal cintilador de NaI (TI) de 3 × 3" oticamente acoplado à fotomultiplicadora, cujo poder de resolução é de $12 \%$ para $60 \mathrm{KeV}$ e de $9 \%$ para $662 \mathrm{KeV}$. Os pulsos gerados são amplificados separadamente e analisados por dois analisadores monocanais e depois contados. A abertura dos canais, para cada caso, é igual ao poder de resolução do detector para aquela energia.

Todas as taxas de contagens obtidas, sofreram correções devido ao tempo morto do sistema (EERRAZ, 1975a), que era da ordem de $5 \times 10^{-6}$ segundos. As taxas de contagens livres de absorvedores ( $I_{0}$ ) eram em torno de $160.000 \mathrm{cpm}$. As frações transmitidas, pro- 
porcionaram taxas de contagens (I) da ordem de 20.000 a 80.000 cpm.

Pelo método $2 \gamma$, as equações utilizadas para a determinação de umidade e densidade são:

$$
\begin{aligned}
& \theta=\frac{\mu_{s a} \cdot L\left(\frac{I_{0}}{I}\right)_{c}-\mu_{s c} \cdot L\left(\frac{I_{0}}{I}\right)_{a}}{x\left(\mu_{s a} \cdot \mu_{w c}-\mu_{s c} \cdot \mu_{w a}\right)} \ldots \ldots \\
& \rho=\frac{\mu_{w c} \cdot L\left(\frac{I_{0}}{I}\right)_{a}-\mu_{w a} \cdot L\left(\frac{I_{0}}{I}\right)_{c}}{x\left(\mu_{s a} \cdot \mu_{w c}-\mu_{s c} \cdot \mu_{w a}\right)}
\end{aligned}
$$

lembrando que os subscritos "a" e "c" se referem, respectivamente, ao ${ }^{241} \mathrm{Am}$ e ao ${ }^{137} \mathrm{Cs}$, e que:

$$
\begin{aligned}
& \text { I = intensidade do feixe que atinge o detector após } \\
& \text { ser atenuado pela amostra (cpm); } \\
& I_{0} \text { = intensidade do feixe que atingiria o detector na } \\
& \text { ausēncia da amostra (cpm); } \\
& x \quad=\text { espessura da amostra }(\mathrm{cm}) \text {; } \\
& \mu_{\text {sa }}=\text { coeficiente de atenuação de massa do solo para } \\
& \text { os raios gama do }{ }^{241} \mathrm{Am}\left(\mathrm{cm}^{2} / \mathrm{g}\right) \text {; } \\
& \mu_{\text {sc }}=\text { coeficiente de atenuação de massa do.solo para } \\
& \text { os raios gama do }{ }^{137} \mathrm{Cs}\left(\mathrm{cm}^{2} / \mathrm{g}\right) \text {; } \\
& \mu_{w a}=\text { coeficiente de atenuação de massa da água para } \\
& \text { os raios gama do }{ }^{241} \mathrm{Am}\left(\mathrm{cm}^{2} / \mathrm{g}\right) \text {; } \\
& \mu_{w c}=\text { coeficiente de atenuação de massa da água para } \\
& \text { os raios gama do }{ }^{137} \mathrm{Cs}\left(\mathrm{cm}^{2} / \mathrm{g}\right) \text {; } \\
& \theta=\text { umidade volumétrica }\left(\mathrm{cm}^{3} \cdot \mathrm{cm}^{-3}\right) \text {; }
\end{aligned}
$$


.22.

$$
\rho=\text { densidade global }\left(\mathrm{g} / \mathrm{cm}^{3}\right) \text {; }
$$

De acordo com FERRAZ (1975b), póde-se estimar as incer tezas nas medidas de densidade e umidade através de (37) e (38), as quais se encontram no Quadro II. 


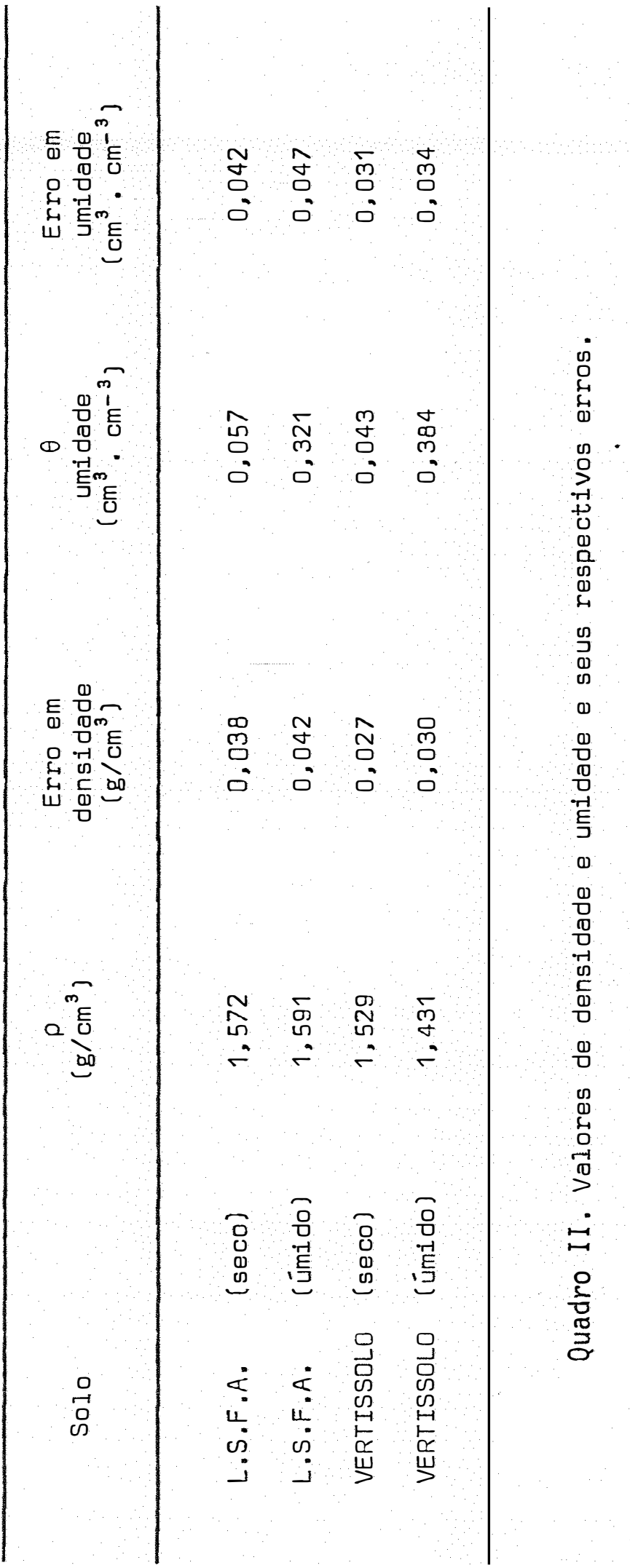


.24 .

\section{RESULTADOS E DISCUSSAO}

\subsection{Obtenção de alguns coeficientes}

Para a utilização do método $2 \gamma$ foi necessário, inicialmente, a obtenção de alguns parâmetros como:

$$
\begin{aligned}
& \theta^{\prime}=\text { umidade inicial do solo seco ao ar; } \\
& \rho_{a}=\text { densidade atual do solo na coluna. }
\end{aligned}
$$

Esses dados e os coeficientes de atenuação de massa obtidos segundo a técnica desenvolvida por FERRAZ (1974), são mostrados no Quadro III. 


\begin{tabular}{ccccc}
\hline Amostra & $\begin{array}{c}\theta^{*} \\
\left(\mathrm{~cm}^{3} \mathrm{~cm}^{-3}\right)\end{array}$ & $\begin{array}{c}\rho_{\mathrm{a}} \\
\left(\mathrm{g} / \mathrm{cm}^{3}\right)\end{array}$ & $\begin{array}{c}\rho_{\mathrm{sa}} \\
\left(\mathrm{cm}^{2} / \mathrm{g}\right)\end{array}$ & $\begin{array}{c}\rho_{\mathrm{sc}} \\
\left(\mathrm{cm}^{2} / \mathrm{g}\right)\end{array}$ \\
\hline 1 & 0,044 & 1,56 & 0,25786 & 0,07669 \\
2 & 0,108 & 1,48 & 0,26857 & 0,07266 \\
3 & 0,029 & 1,38 & 0,29598 & 0,07618 \\
4 & 0,021 & 1,38 & 0,29835 & 0,07677 \\
5 & 0,055 & 1,50 & 0,26568 & 0,07216 \\
$\bar{A}$ gua & - & - & 0,20601 & 0,08754 \\
\hline
\end{tabular}

Quadro III. Valores dos coeficientes de cada amostra, necessários à utilização do método $2 \gamma$.

\subsection{Valores de a e expansão do solo}

Para cada amostra, observou-se a expansão total do solo dentro da coluna, na extremidade de admissão da água, o que é mostrado no Quadro IV. No mesmo Quadro, podem ser vistos os valores do parámetro a bem como os coeficientes de correlação $(r)$ das retas (32), para as referidas amostras. Não houve, pelo que pode ser obser vado, uma dependéncia marcante entre a expansão do solo e o fato do parámetro a diferir de 0,50. No entanto, para os dois casos em que se verificou uma grande expansão ( 1 e 5 ), obteve-se os valores mais baixos para a. Percebeu-se também a possibilidade de a ser diferente de 0,50, mesmo para solos que não apresentam, praticamente, expansão, como aconteceu para a amostra 4 , e também para os dados de QUEIROZ (1976), cujos valores de a calculados são geralmente inferiores a 0,50. Nesses casos, parece saliente o fato de haver um rearranjo interno significativo das partículas do solo, apesar de não haver uma expansão visível. Portanto, tudo leva a crer que esse rearranjo das 
partículas, é um fenōmeno mais importante que a própria expansão,pois estando sempre presente, está constantemente atuando sobre o fluxo. 0 próprio potencial matricial, $\xi$, considerado inicialmente apenas função da umidade, $\theta$, deve conter também uma dependéncia com o tempo, $t$ (ou com a posição, $\chi$ ), já que $\xi$ é o resultado de forças de interação entre a água e as partículas do solo. Assim, a formulação da função $\xi=\xi(\theta, t)$ é mais adequada do que a anterior, $\xi=\xi(\theta)$, pois inclui a dependência com o tempo, e pode ser usada para qualquer tipo de solo. Essa formulação também permite um estüdo mais aprofundado de outros fenomenos, como é o caso da histerese. É interessante reforçar o exposto em (33), isto é, quando $a=0,50, \Omega=t^{*}$, que explica o caso dos solos onde o rearranjo é despresivel, e portanto a dependência do tempo, acima sugerida, não é necessária.

\begin{tabular}{cccc}
\hline Amostra & $\begin{array}{c}\text { Parámetro } \\
\text { do solo } \\
a\end{array}$ & $\begin{array}{c}\text { Coeficiente } \\
\text { de correlaçäo } \\
(\boldsymbol{r})\end{array}$ & $\begin{array}{c}\text { Expansão } \\
\text { do solo } \\
\text { (mm) }\end{array}$ \\
\hline 1 & 0,43 & 0,99891 & 3,0 \\
2 & 0,50 & 0,99961 & 1,5 \\
3 & 0,50 & 0,99622 & 2,0 \\
5 & 0,45 & 0,99854 & 0,5 \\
\hline
\end{tabular}

Quadro IV. Valores do parámetro $a$, do coeficiente de correlação da reta (32), e da expansão total da amostra de solo.

Analisando-se os dados de REICHARDT e NIELSEN (1975), que trabalharam com solo expansivo, obtém-se $a=0,42$, o mesmo acon- 
tecendo com outros trabalhos similares, onde os valores de a oscilam entre 0,38 e 0,50 . Isso implica em $D_{2}(t)<1$, o que faz com que $D(\theta, t)$ seja uma função decrescente no tempo ou, no caso limite em que $a=0,50$, constante em relação ao tempo.

No gráfico da figura II, são mostradas as retas obtidas com os dados de cada amostra, de acordo com a equação (32). Convem notar os diferentes valores de $a$ calculados, bem como os de $\lambda_{6}$. Os pares de valores $\left(a, \lambda_{f}\right)$ podem ser vistos como uma característica das amostras de solo, característica essa que deve favorecer o estudo da generalização da infiltração da água no solo.

\subsection{Perfis de umidade e difusividade}

Através do método $2 \gamma$, foram obtidos os perfis de umi dade que se encontram nas figuras de III a VII. 0 número de perfis de umidade para cada coluna variou de 3 a 5, de acorco com a rapidez de infiltração da água em cada uma. O valor mais baixo de umidade é sempre a umidade inicial do solo seco ao ar $\left(\theta^{\prime}\right)$ e o valor mais alto é, praticamente, a umidade de saturação $\left(\theta_{s}\right)$. Esses valores limi tes foram obtidos, seguindo a técnica utilizada por LIBARDI (1973).

A partir desses perfis de umidade, pôde-se calcular a difusividade da água do solo para cada amostra, utilizando-se do méto do de BRUCE e KLUTE (1956). Nos Quadros de V a VIII, estão os valores de difusividades para as respectivas amostras, onde pode-se obser var a diferença entre os dados obtidos em tempos distintos, bem como compará-los cóm o valor de $D^{\star}(\theta)$ que é o valor da difusividade obtido em se considerando a mesma apenas função da umidade. Apesar de $D(\theta, t)$ e $D^{\star}(\theta)$ apresentarem a mesma ordem de grandeza, nota-se a grande diferença entre $D_{1}(\theta)$ e $D^{\star}(\theta)$, e o fato deste último poder ser dado como um $D(\theta, t)$, para o qual o tempo pode ser facilmente calculado através de (11) e (25). Para as amostras 2 e 3 , que tem $a=0,50$, as difusividades são apenas função da umidade, de acordo 
com (35), como pode ser visto no Quadro VI. A amostra 5, que apresen tou o valor de a mais baixo, tem seus valores de difusividade mostrados no Quadro VIII, onde se pode ver claramente a diferença entre os dados obtidos em tempos distintos.

\subsection{Liberdade de expansão para os solos}

Através do Quadro IV, pode-se notar que os valores de expansão do solo são pequenos em comparação ao tamanho normal da mola, e assim também as forças de restauração, que podem ser.calculadas por (36). Aparentemente, a mola não influi na liberdade de expansão do solo.

De acordo com LAL et alii (1970), os efeitos de expansão de solos em colunas se tornam bem mais visíveis quando o diàmetro interno da coluna é maior que $10 \mathrm{~cm}$. Neste caso, foi usada uma coluna de $5,65 \mathrm{~cm}$ de diámetro interno, não só pelas dificuldades em se ob ter outras colunas com a medida acima referida, como também para poder haver uma comparação dos resultados deste trabalho com dados já existentes, obtidos nas mesmas colunas, por outros autores, como REICHARDT e NIELSEN (1975), LIBARDI (1973), QUEIROZ (1976), etc..

\subsection{Método e arranjo experimental}

O arranjo experimental utilizado provou ser adequado para esse tipo de estudo, já que permitiu medir a expansão das amostras de solo durante a infiltração da água, como era um dos objetivos iniciais deste trabalho. Para isso, contribuiram a pequena força de restauração da mola aos deslocamentos observados e o pequeno atrito entre o pistão e as paredes da coluna. Também a utilização do método $2 \gamma$ foi plenamente justificada, já que com apenas um feixe de radiação gama não seria possível determinar as variações de densidade das

amostras de solo nos pontos onde ocorreram expansões. O gráfico da 
Figura VIII, elaborado com os dados obtidos com a amostra 5, serve pa ra evidenciar esse fato. Observe-se que um aumento de umidade, de 0,35 a $0,50 \mathrm{~cm}^{3} \cdot \mathrm{cm}^{-3}$, pode ocasionar uma diminuição aproximada de $10 \%$ em densidade para esse tipo de solo, como já foi mastrado por EERRAZ e GUERRINI (1975), em trabalho preliminar. 


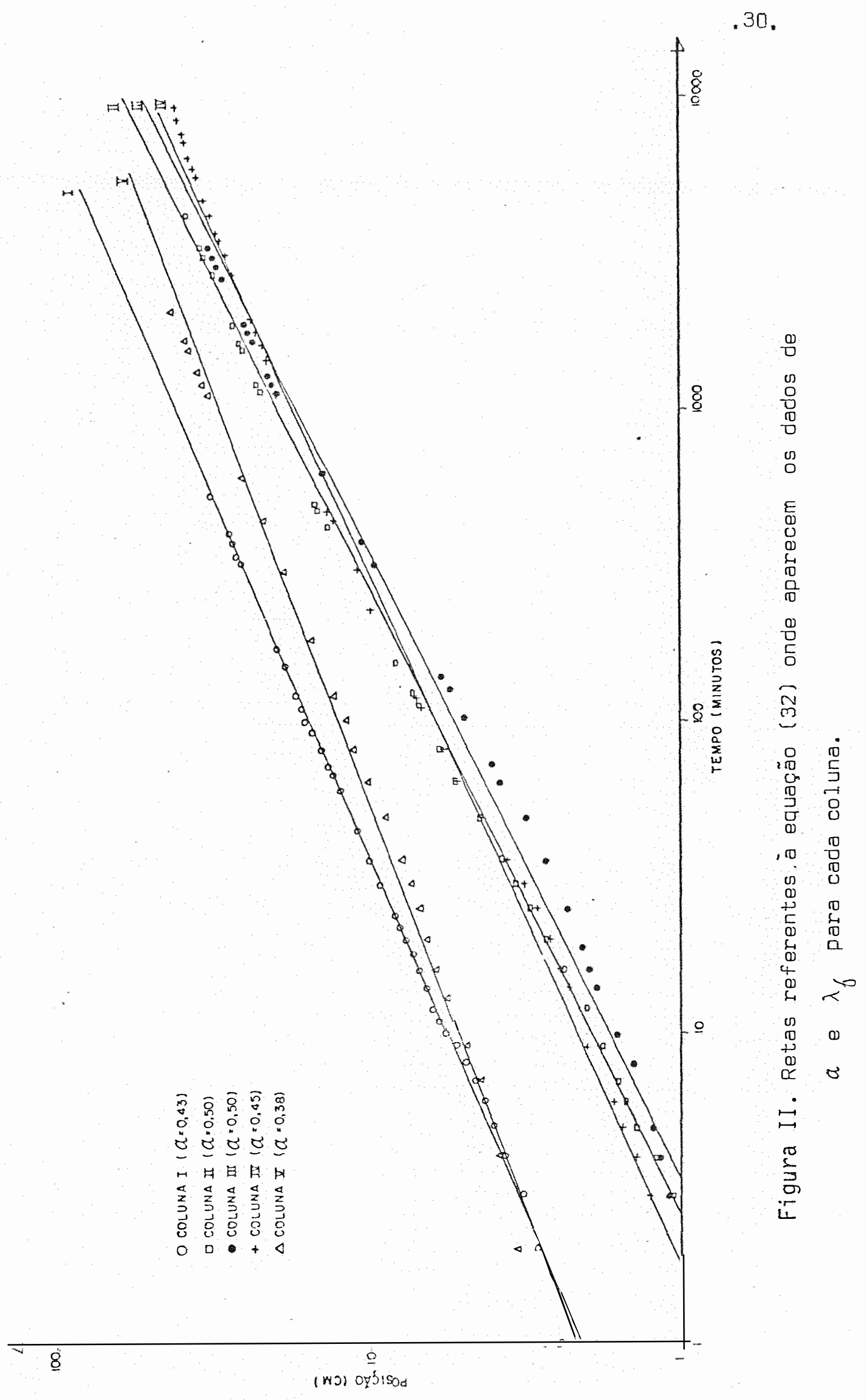




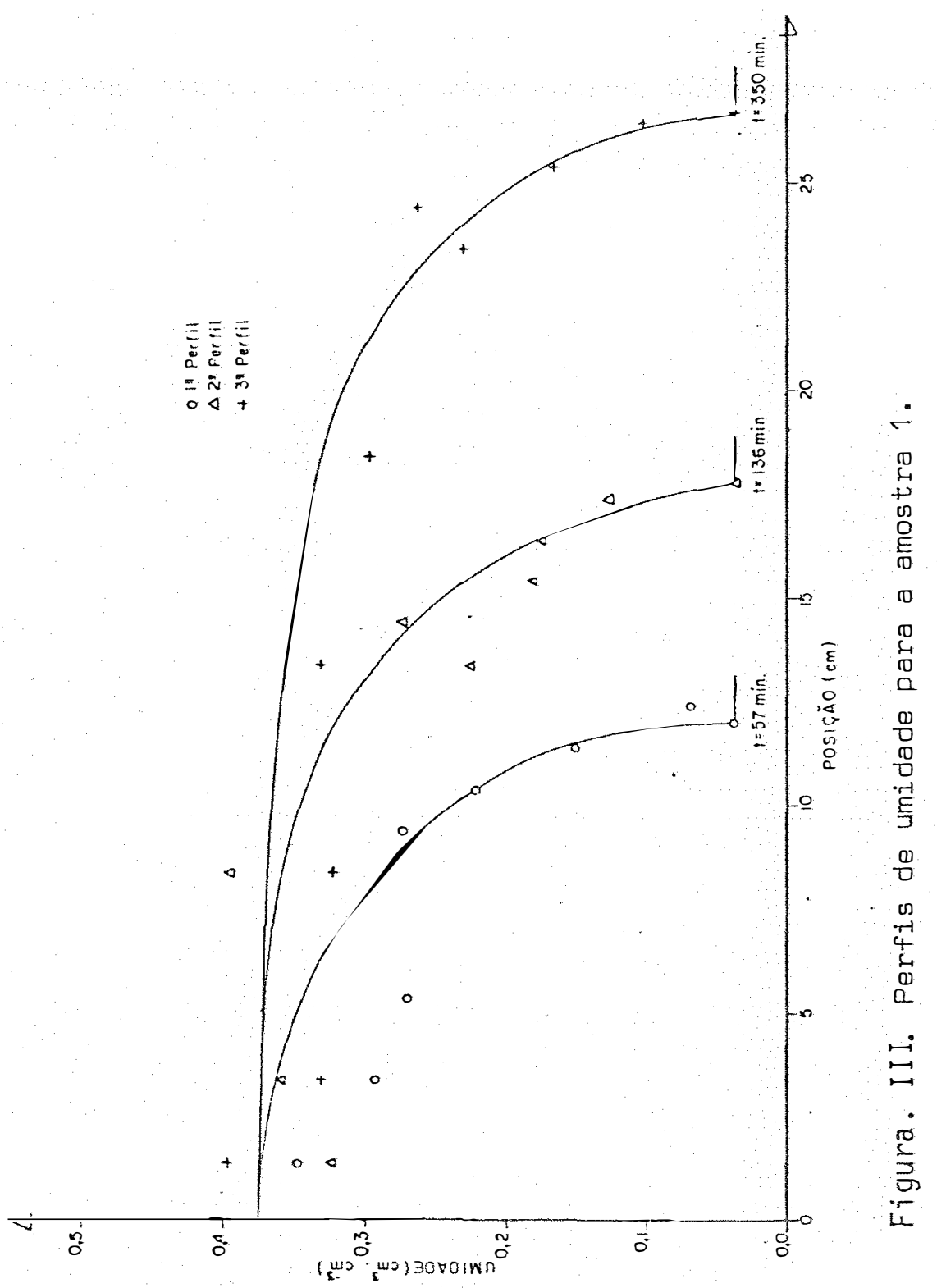




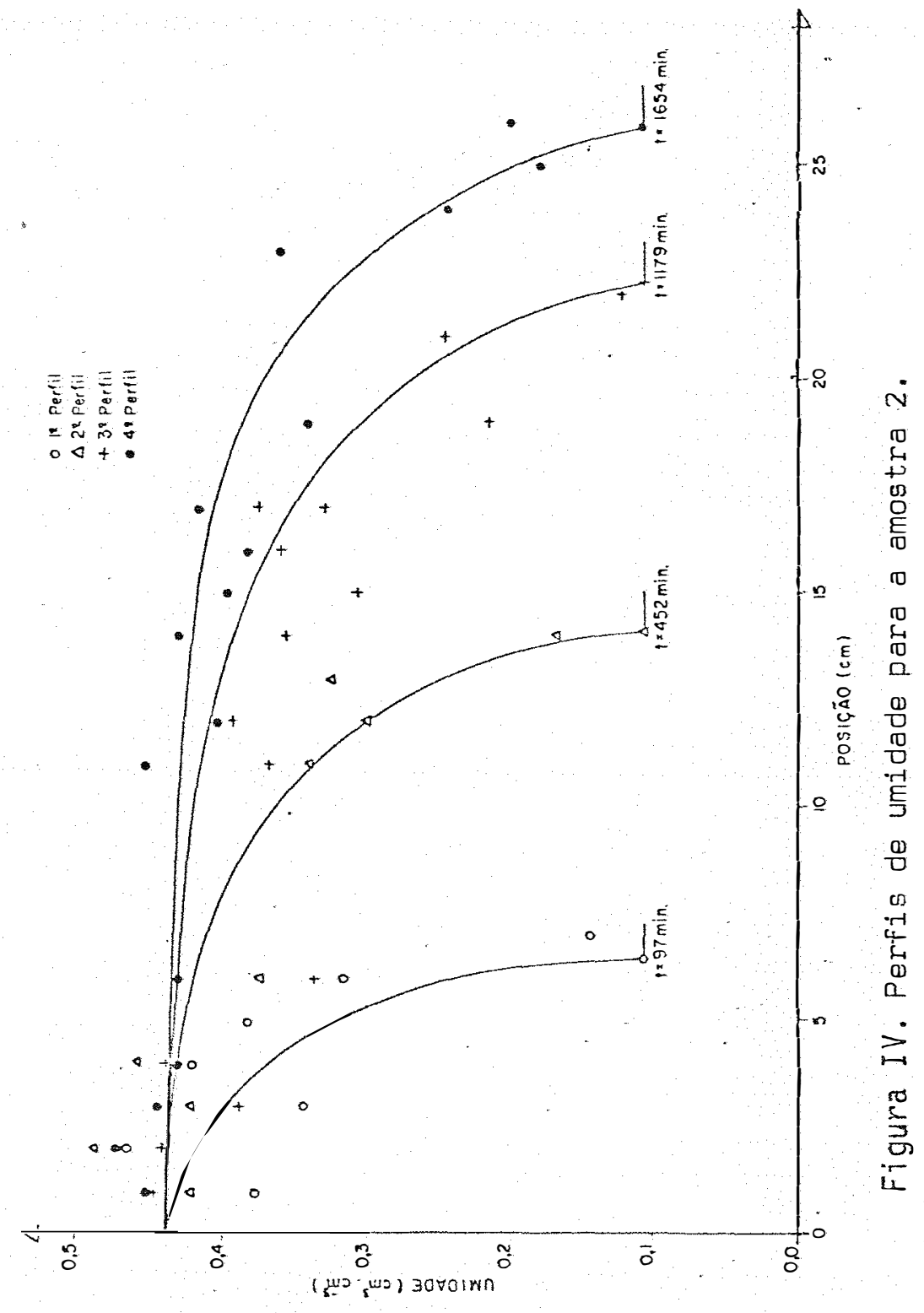


.33.

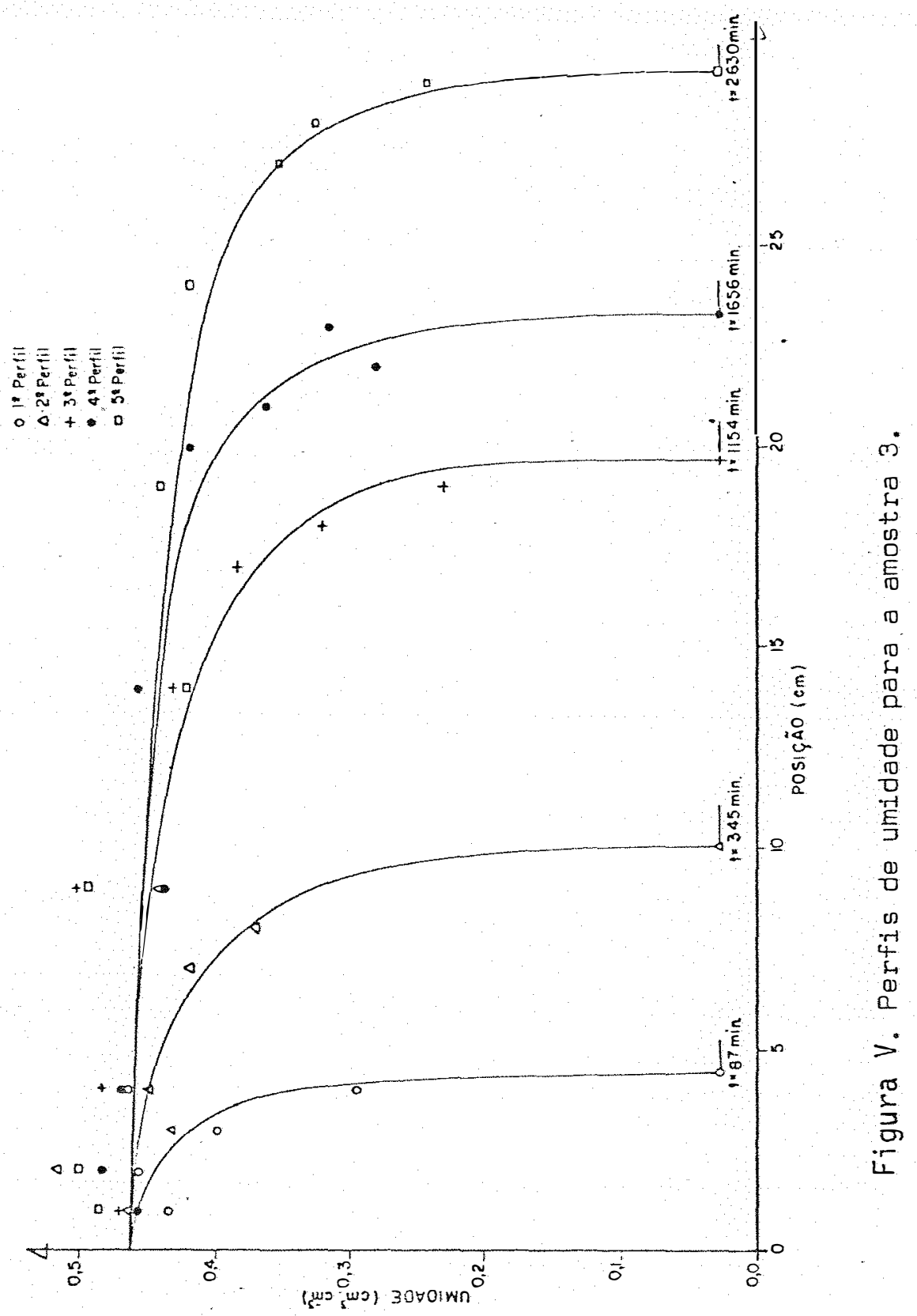


.34.

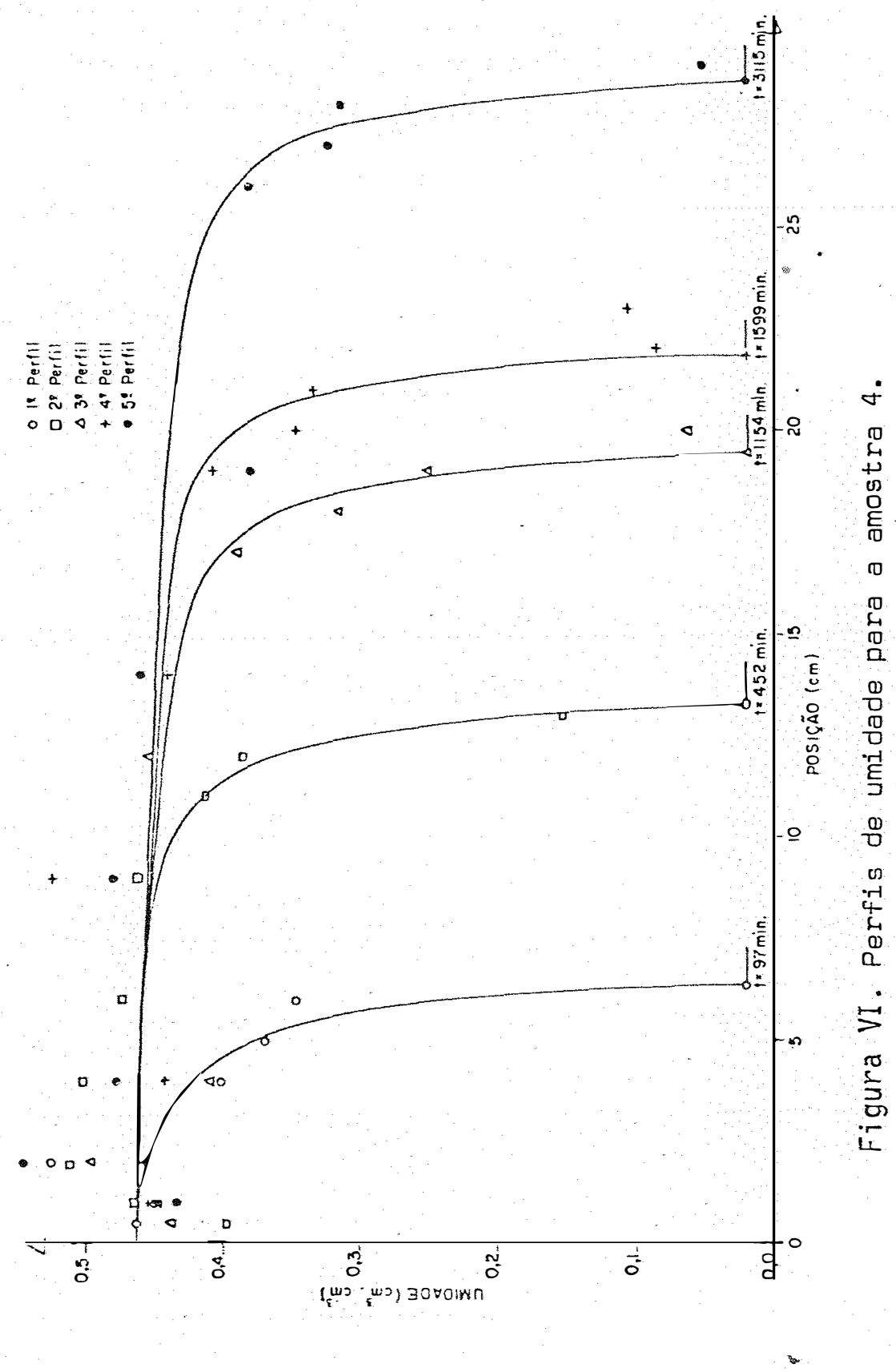


.35.

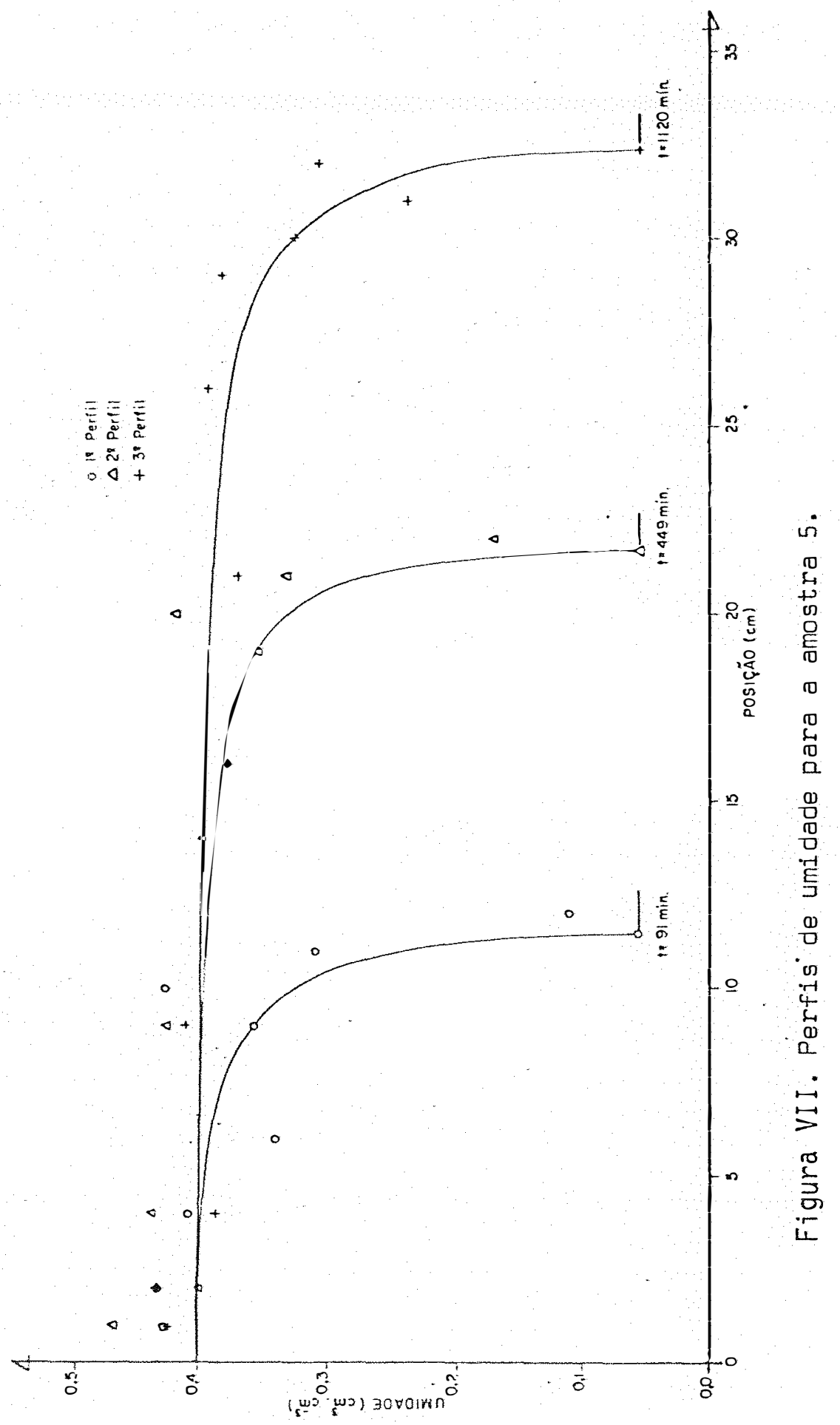


.36 .

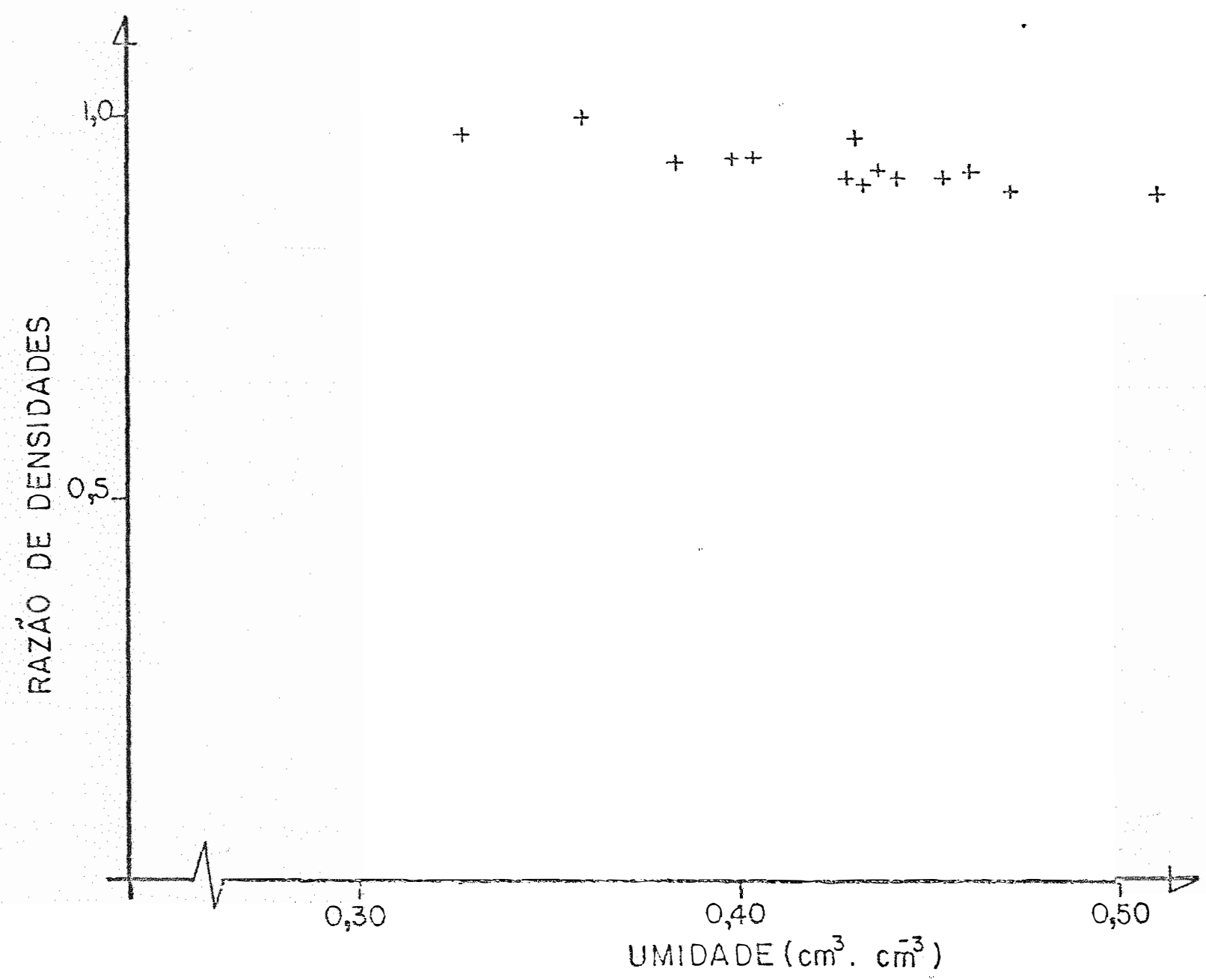

Figura VIII. Razão entre as densidades referentes aos valores de umidades correspondentes e a densidade média da coIuna seca, em função da umidade. 
.37.

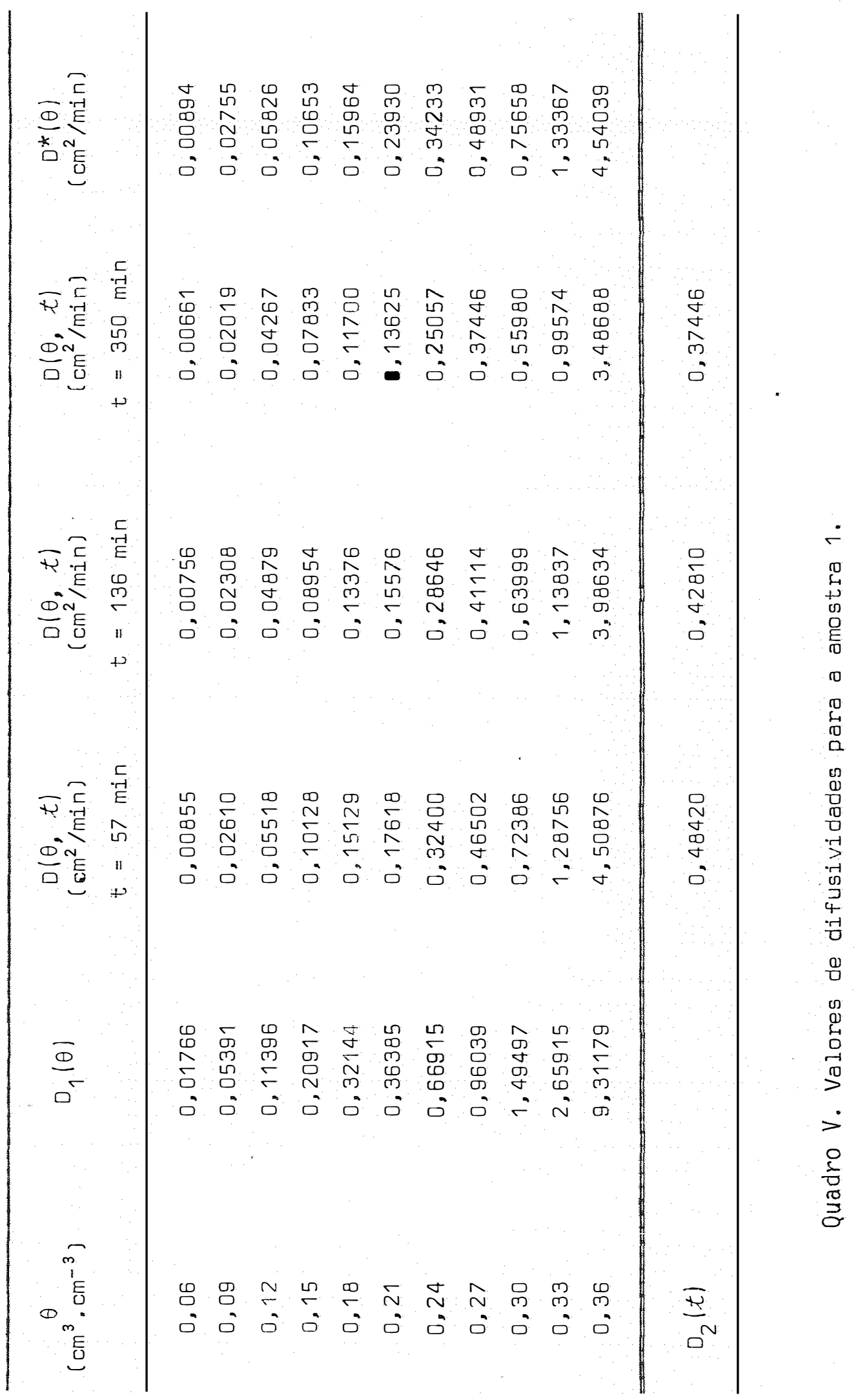


.38 .

\begin{tabular}{|c|c|c|}
\hline$\left(\mathrm{cm}^{3} \cdot \mathrm{cm}^{-3}\right)$ & $\begin{array}{c}D(\theta, t) \\
\left(\mathrm{cm}^{2} / \mathrm{min}\right\} \\
\text { amostra } 2\end{array}$ & $\begin{array}{c}D(\theta, t) \\
\left(\mathrm{cm}^{2} / \mathrm{min}\right) \\
\text { amostra } 3\end{array}$ \\
\hline 0,06 & - & 0,00068 \\
\hline 0,09 & - & 0,00203 \\
\hline 0,12 & 0,00100 & 0,00338 \\
\hline 0,15 & 0,00475 & 0,00537 \\
\hline 0,18 & 0,01078 & 0,00785 \\
\hline 0,21 & 0,01887 & 0,01122 \\
\hline 0,24 & 0,03165 & 0,01599 \\
\hline 0,27 & 0,04981 & 0,02237 \\
\hline 0,30 & 0,07440 & 0,03262 \\
\hline 0,33 & 0,10474 & 0,05024 \\
\hline 0,36 & 0,16075 & 0,08209 \\
\hline 0,39 & 0,27102 & 0,14034 \\
\hline 0,42 & 0,79526 & 0,25126 \\
\hline 0,45 & - & 1,10347 \\
\hline
\end{tabular}

Quadro VI. Valores de difusividades para as amostras 2 e 3 , os quais não variam no tempo, e portanto, $0(\theta, t) \equiv D_{1}(\theta) \equiv 0^{*}(\theta)$, já que essas amostras apresentaram $a=0,50$ 


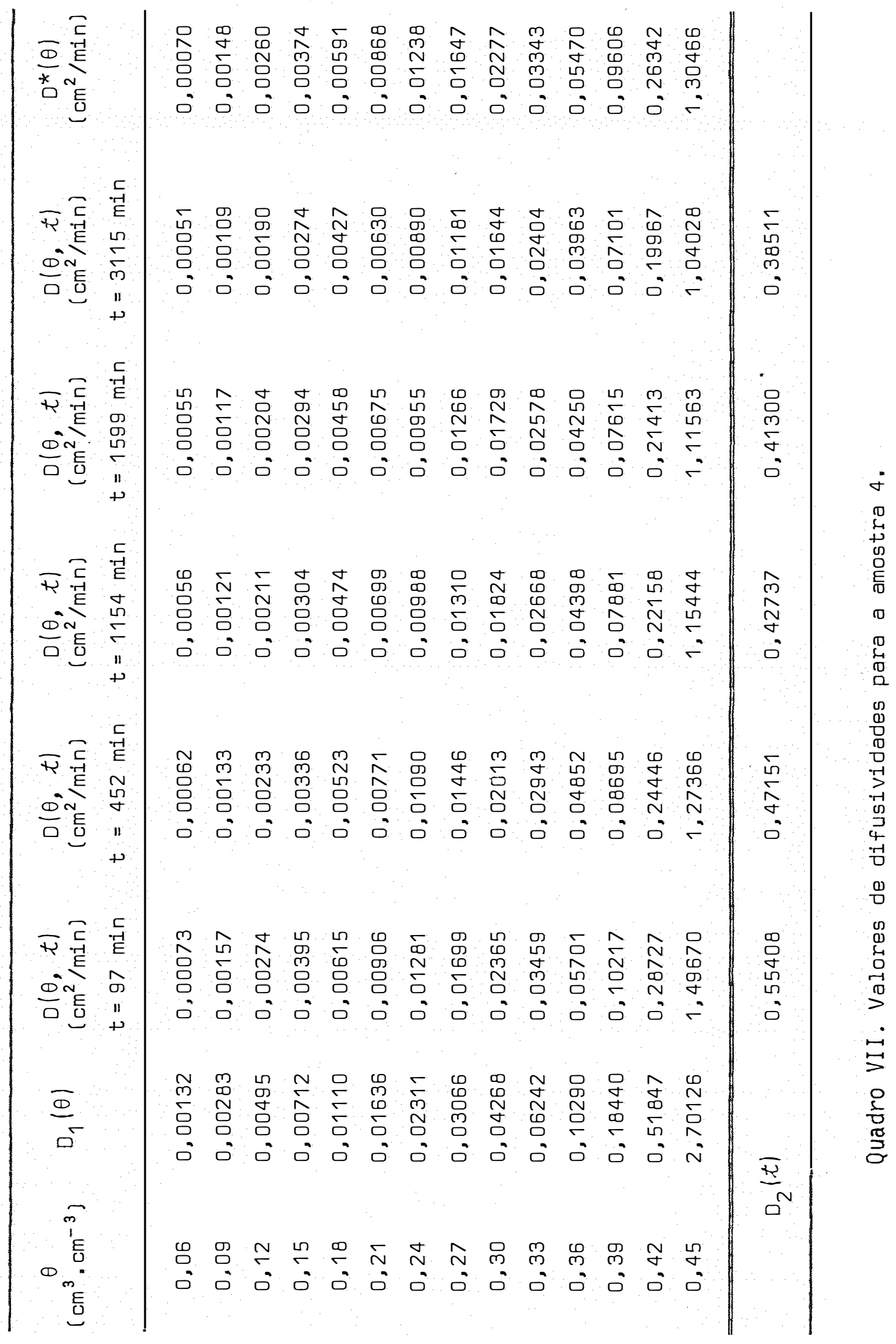




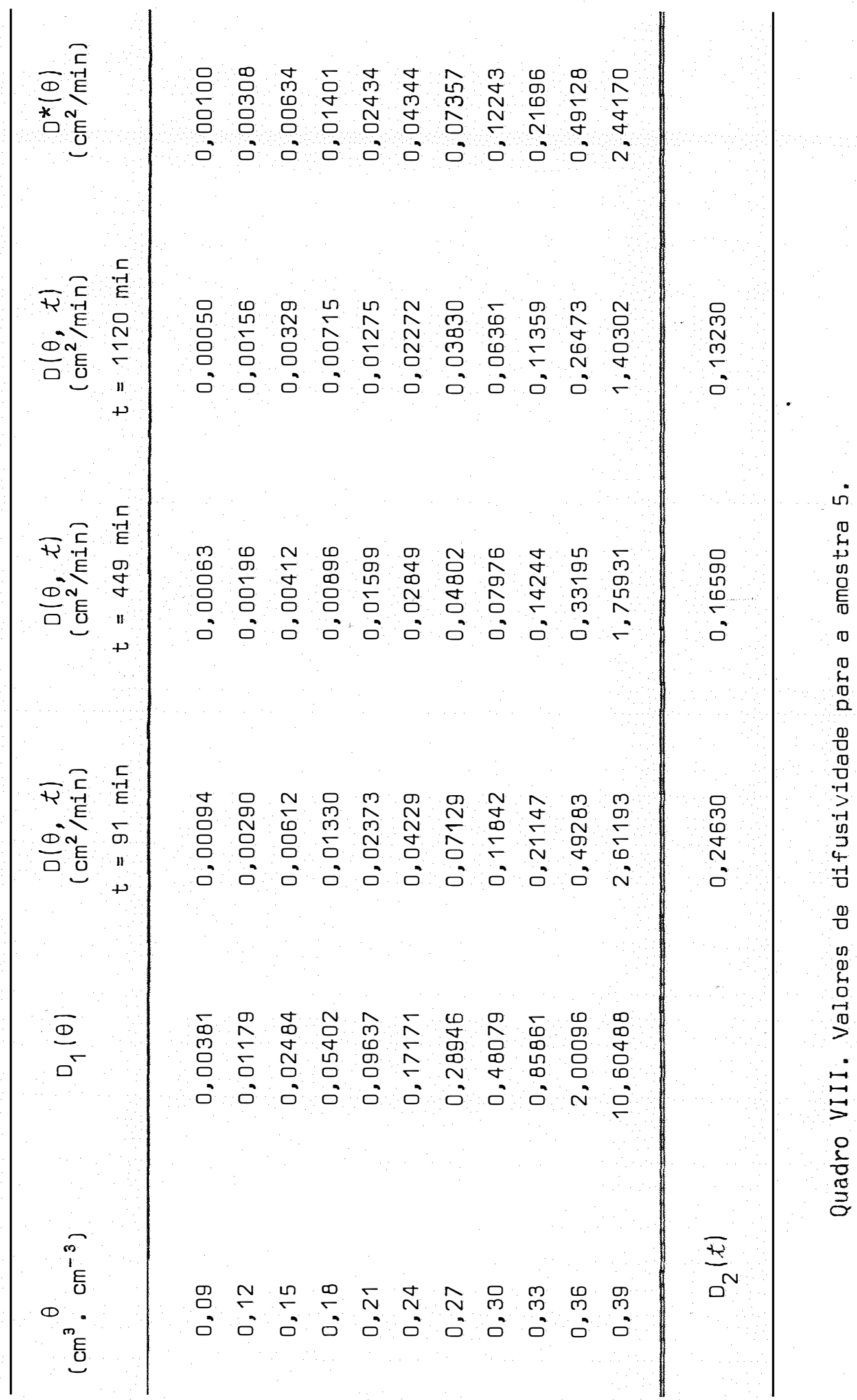


.41.

\section{CONCLUSOEES}

1. O rearranjo interno das partículas do solo à passagem da água, é um fenômeno significativo em determinados tipos de so10, influindo, nesses casos, no movimento da água através do solo.

2. Esse fenómeno, embora ocorra também em solos expansivos, não apresenta correlação direta com a expansão, sendo portanto, fenómenos distintos.

3. Em decorréncia do fenómeno do rearranjo interno das partículas, a difusividade da àgua do solo é mais precisamente estima da considerando-a como uma função da umidade e do tempo.

4. Apenas quando esse rearranjo é desprezível, a difu- 
sividade pode ser dada únicamente como função da umidade.

5. A difusividade, comumente calculada através de BRUCE e KLUTE (1956), na verdade é uma função da umidade e do tempo, para a qual este último pode ser calculado.

6. O rearranjo interno das partículas ainda sugere que $\xi=\xi(\theta, t)$ seja uma formulação mais adequada que $\xi=\xi(\theta)$, inclusi ve trazendo mais luz para o estudo do fenómeno da histerese.

7. Os valores de a encontrados na parte experimental deste trabalho, bem como os calculados com dados de trabalhos de outros autores, se situam dentro dos limites de 0,38 a 0,50.

8. Como não foram encontrados valores de a superiores a 0,50, isto significa que, dentre os experimentos realizados e anali zados, a difusividade é decrescente no tempo, ou, no caso limite de $a=0,50$, ela é constante no tempo.

9. Os pares de valores $(a, \lambda f)$ servem para caracteri zar os tipos de solo, no estudo da generalização da infiltração da água no solo.

10. A montagem experimental, com o pistão e a mola dentro da coluna, mostrou-se adequada, assim como o método $2 \gamma$ que permi tiu a determinação das variações de densidade das amostras de solo à passagem da água. 
A difusividade da água do solo é estudada como sendo função da umidade e do tempo. A partir da idéia de se estudar o movi mento horizontal da água em solos expansivos, é obtida uma formulação simples que permite o cálculo da difusividade, função da umidade e do tempo, não só nesse tipo de solo mas em qualquer outro. Observou-se que o rearranjo interno das partículas do solo é um fenômeno mais importante que a própria expansão, sendo responsável pela dependéncia do tempo para a difusividade. Foi empregado o método $2 \gamma$, com o qual é possível a determinação simultánea de densidade e umidade, ponto por ponto, numa coluna de solo. Compararam-se os valores de difusividade assim obtidos com aqueles alcançados, quando não se considera a depen dência do tempo. Finalmente, um novo parámetro do solo, $a$, é introdu zido e seus valores obtidos confirmam a hipótese do rearranjo e da de pendência do tempo para a difusividade. 
.44 .

\section{SUMMARY}

The soil-water diffusivity has been studied as a function of water content and time. From the idea of studying the horizontal movement of water in swelling soils, a simple formulation has been achieved which allows for the diffusivity, water content dependency and time dependency, to be estimated, not only for this kind of soil, but for any other soil as well. It was observed that the internal rearrangement of soil particles is a more important phenomenon than swelling, being responsible for time dependency. The method $2 \gamma$ was utilized, which makes it possible to simultaneously determine the water content and density, point by point, in a soil column. The diffusivity data thus obtained were compared to those obtained when time dependency is not considered. Finally, a new soil parameter, $a$, is introduced and the values obtained agrees with the internal rearrangement assumption and time dependency for diffusivity. 


\section{BIBLIOGRAFIA}

AHUJA, L.R., 1975. A one-step wetting procedure for determing both water caracteristic and hydraulic conductivity of a soil core. Soil Sci. Soc. American Proceedings, 39:418-23.

AHUJA, L.R. e D.SWARTZENDRUBER, 1972. An improved form of soil-water diffusivity function. Soil Sci. Soc. American Proceedings, 36: 914 .

BRIDGE, B.J. e N.COLLIS-GEORGE, 1973a. An experimental study of vertical infiltration into a structurally unstable swelling soil, with particular reference to the infiltration throttle. Aust. J. Soil Res., 11: 121-32. 
BRIDGE, B.J. e N.COLLIS-GEORGE, 1973b. A dual source gamma ray traversing mechanism suitable for the non-destructive simultaneous measurements of bulk density and water content in collumns of swelling soil. Aust. J. Soil Res., 11: 83-92.

BRIDGE, B.J.; N.COLLIS-GEORGE e R.LAL, 1970. The effect of wall lubricants and column confinement on the infiltration behavior of a swelling soil in the laboratory. Aust. J. Soil Res., 8: 25972.

BRIONES, A.A. e G.UEHARA, 1969. Water movement in shrinking soils. Universidade do Hawai (Tese de Doutoramento).

BRUCE, R.R. e A.KLUTE, 1956. Measurement of soil moisture diffusivity. Soil Sci. Soc. American Proceedings, 20: 458-62.

COLLIS-GEORGE, N. e R.LAL, 1970. Infiltration into columns of swelling soil as studied by high speed photography. Aust. J. Soil Res., 8: 195-207.

COLLIS-GEORGE, N.e B.J.BRIDGE, 1973. The effect of height of sample and confinement on the moisture characteristic of an aggregated swelling clay soil. Aust. J. Soil Res., 11:107-20.

COUGHLAN, K.J.; W.E.FOX e J.D. HUGHES, 1973. Aggregation in swelling clay soils. Aust. J. Soil Res., 11: 133-41.

DOLEZAL, F. e M.KUTILEK. 1972. Flow of water in swelling soil. Proceedings of the second symposium on fundamentals of transport phenomena in porous media. vol. 1. University of Guelph, Dntario, Canadá.

EL-SWAIFY, S.A. e D.W.HENDERSON, 1967. Water retention by osmotic swelling of certain colloidal clays with varying ionic composition. J. Soil Science, 18(2): 223-32. 
EERRAZ, E.S.B., 1974. Determinação simultànea de densidade e umidade de solos por atenuação de raios gama do ${ }^{137} \mathrm{Cs}$ e ${ }^{241} \mathrm{Am}$. Piracicaba, ESALQ/USP, $120 \mathrm{p}$ (Tese de Livre-Docência).

FERRAZ, E.S.B., 1975a. Influênci a do tempo morto nas determinações de densidade e umidade de solos por atenuação gama. In: III Encon tro sobre Escoamento em Meios Porosos, Maringá- PR.

FERRA2, E.S.B., 1975b. "Espessura da amostra em densimetria de solos por atenuação de radiação gama. In: III Encontro sobre Escoamento em Meios Porosos, Maringä-PR.

FERRAZ, E.S.B.; M.L. ALMEIDA e I.A.GUERRINI, 1974. Uso de uma fonte de radiação gama de $60 \mathrm{KeV}$ para determinação da umidade e densidade de solos. In: Reunião da Sociedade Brasizeira para o Progresso da Ciência, 26à., Recife, PE.

FERRAZ, E.S.B. e I.A.GUERRINI, 1975. Emprego do método $2 \gamma$ no estu do de solos expansivos (swelling soils). In: III Encontro sobre Escoamento em Meios Porosos, Maringā-PR.

FOX, W.E., 1964. A study of bulk density and water in a swelling soil. Soil Science, 98: 307-16.

JONG, E., 1966. Hydraulic conductivity of clays during shrinkage. Soil Sci. Soc. American Proceedings, 30 (3): 289-92.

LAL, R.; B.J.BRIDGE e N.COLLIS-GEORGE, 1970. The effect of column diameter on the infiltration behaviour of a swelling soil. Aust. J. Soil Res., 8: 185-93.

LAROUSSI, C.; G.VANDERVOORDE e L.DE BACKER, 1975. Experimental investigation of the diffusivity coefficient. Soil Sciences, 120 (4): $249-55$.

LIBARDI, P.L., '1973. Infiltração da água no solo: uma generalização. Piracicaba, ESALQ/USP, 46 p. (Tese de Mestrado). 
OLESEN, S.E., 1973. Gamma radiation for measuring water contents in soils columns with changing bulk density. J. Soil Science, 24(24) : 461-9.

PARLANGE, J.Y., 1973. Horizontal infiltration of water in soils: a theorical interpretation of recent experiments. Soil Sci. Soc. American Proceedings, 37: 329-30.

PARLANGE, J.Y., 1975a. A note on the moisture diffusivity of saturated swelling systems from desorption experiments. Soil Science, 120 (2): 156-8.

PARLANGE, J.Y., 1975b. On solving the flow equation in unsaturated soils by optimization: horizontal infiltration. Soil Sci. Soc. American Proceedings, 39: 415-7.

PHILIP, J.R., 1968. Kinetics of sorption and volume change in claycolloid pastes. Aust. J. Soil. Res., 6: 249-67.

PHILIP, J.R., 1969a. Hydrostatics and hydrodynamics in swelling média. International Association of Hydraulic Research Symposium on Fundamentals of Transport Phenomena in Porous Media. ISARAEL.

PHILIP, J.R., 1969b. Moisture equilibrium in the vertical in swelling soils: I - Basic Theory. Aust. J. Soil Res., 7: 99-120.

PHILIP, J.R., 1969c. Moisture equilibrium in the vertical in swelling soils: II - Applications. Aust. J. Soil Res., 7: 121-41. PHILIP, J.R., 1970. Hydrostatics in swelling soils and soil suspensions: unification of concepts. Soil Science, 109 (5): 2948.

PHILIP, J.R. e D.E.SMILES, 1969. Kinetics of sorption and volume change in three-component systems. Aust. J. Soil Res., 7: 1-19. 
QUEIROZ, E.N., 1976. Movimento da agua em solos dos tabuleiros do Nordeste. Piracicaba, ESALQ/USP, 57 p. (Tese de Mestrado).

RAATS, P.A.C. e A.KLUTE, 1968. Transport in soils: the balance of mass. Soil Sci. Soc. American Proceedings, 32 (2): 161-6.

REGINATO, R.J., 1974. Water content and bulk density changes in a soil pedon measured with dual energy gamma-ray transmission. Can. J. Soil Sci. 54: 325-8.

REICHARDT, K., 1976. Processos de transferéncia no sistema soloplanta-atmosfera. Fundação CARGILL/CENA/USP.

REICHARDT, K.; D.R.NIELSEN e J.W.BIGGAR, 1972. Scaling of horizontal infiltration into homogeneous soils. Soil Sci. Soc. American Proceedings, 36: 241-5.

REICHARDT, K. e P.L.LIBARDI, 1973. A new equation to estimate soilwater diffusivity. In: FAO/IAEA Symposium on Isotopes and Radiation Techniques in Studies of Soil Physics, Irrigation and Drainage in Relation to Crop Production. Vienna, Austria.

REICHARDT, K. e D.R.NIELSEN, 1975. Infiltration into swelling soils. In: III Encontro sobre Escoamento em Meios Porosos, Maringä-PR.

SMILES, D.E. e M.J.ROSENTHAL, 1968. The movement of water in swelling materials. Aust. J. Soil Res., 6: 237-48.

SMILES, D.E. e H.G.POULOS, 1969. The one-dimensional consolidation of columns of soil of finite lenght. Aust. J. Soil Res., 7: 28591.

SMILES, D.E. e A.G.HARVEY, 1973. Measurement of moisture diffusivity of wet swelling systems. Soil Science, 116(6): 391-9. 
.50 .

SOANE, B.D., 1967. Dual energy gamma ray transmission for coincident measurement of water content and dry bulk density in soil. Nature, 241: 1273.

SPOSITO, G., 1973. Volume changes in swelling clays. Soil Sci. 115 (4): 315-20.

SPOSITO, G., 1975. Steady vertical flows in swelling soils. Water Resour. Res. 11 (3): 461-4.

STROOSNIJDER, I. e J.G. DE SWART, 1974. Columin scanning with simultaneous use of ${ }^{241} \mathrm{Am}$ and ${ }^{137} \mathrm{Cs}$ gamma radiation. Soil Science. $118(2): 61-9$.

SWARTZENDRUBER, D., 1969. The flow of water in unsaturated soils in "Flow through Porous Media". Academic Press. New York.

TALSMA, T., 1974. Moisture profiles in swelling soils. Aust. J. Soil Res., 12: 71-5.

YONG, R.N. e B.P.WARKENTIN, 1972. Unsaturated flow in expansive soils. Proceedings of the second symposium on fundamentals of transport phenomena in porous media. vol. 1. University of Guelph, Ontario, Canadá.

WHISLER, F.D.; A.KLUTE e D.B.PETERS, 1968. Soil water diffusivity from horizontal infiltration. Soil Sci. Soc. American Proceedings, 32: 6-11.

WONG, H.Y. e R.N. YONG, 1975. Unsaturated flow mechanism in lowswelling clays. Soil Science, 120(5): 339-48.

ZASLAVSKI, $\dot{D} .$, 1964. Saturated and unsaturated flow equation in an unstable porous medium. Soil Science, 98: 317-21. 